\title{
9. MINERALOGIC AND PETROGRAPHIC RECORDS OF SEDIMENT-FLUID INTERACTION IN THE SEDIMENTARY SEQUENCE AT MIDDLE VALLEY, JUAN DE FUCA RIDGE, LEG $139^{1}$
}

\author{
Martine D. Buatier, ${ }^{2}$ Anne-Marie Karpoff, ${ }^{3}$ Maria Boni, ${ }^{4}$ \\ Gretchen L. Früh-Green, ${ }^{5}$ and Judith A. McKenzie ${ }^{5}$
}

\begin{abstract}
Petrographic and mineralogic studies of the sedimentary cover drilled at Middle Valley on the Juan de Fuca Ridge allow characterization of the newly formed silicate and carbonate phases and a determination of their origin. At all drill sites, the sediments are composed of a mixture of detrital and authigenic minerals in various proportions. The unmodified sediments forming the surficial cover consist of detrital minerals, including quartz, feldspar, amphibole, mica, clays (smectite, chlorite), and biogenic remains. Quartz, feldspar, and mica are still present in hydrothermally modified sediments where they coexist with newly formed minerals, except in Hole 858B, which is located close to an active hydrothermal vent where pure hydrothermal layers consist solely of authigenic minerals.

The main authigenic phases in the modified sediments are silicate minerals and calcite concretions with minor sulfides. The most common authigenic silicate in the deepest layers downhole is $\mathrm{Mg}$-chlorite. At Site 858, a mineralogic sequence is clearly established from the seawater/sediment interface to the deeper parts of the hole. The shallowest hydrothermal unit from Hole $858 \mathrm{~B}$ is composed of $\mathrm{Mg}$-smectite and authigenic pyrite, which downhole sequentially overlies corrensite-rich layers which contain some talc and anhydrite. In the deepest unit, chlorite is the dominant phyllosilicate coexisting with authigenic quartz. All of these silicates seem to be the product of a direct precipitation from hydrothermal fluids. This sequence reflects a thermal gradient related to the vertical influx of hydrothermal fluids.

Secondary precipitation of carbonate concretions was generated from Ca released by biogenic phases and hydrothermal fluids. The concretions show several stages of crystallization and seem to occur preferentially in oxidizing areas and in the shallowest deposits. The occurrence of the concretions is favored by high porosity in the sediments.

The presence of pure hydrothermal layers locally interbedded with unmodified sediments, and the variable mineralogy of the carbonate nodules suggest that convective circulation of fluids acts at various scales in this hydrothermal system and that the sedimentary cover favors lateral fluid circulation.
\end{abstract}

\section{INTRODUCTION}

Middle Valley on the northern Juan de Fuca Ridge is one of a few well-studied sediment-covered spreading centers in the eastern $\mathrm{Pa}$ cific Ocean and was the subject of detailed investigations during Ocean Drilling Program Leg 139 (Davis and Villinger, 1992; Davis, Mottl, Fisher et al., 1992). Four sites were drilled in the eastern part of the valley during Leg 139. Each of the sites is characterized by a distinct hypothetical hydrologic environment within the Middle Valley rift. These environments range from an area of fluid recharge at lower temperatures (Site 855), to an area of active discharge characterized by high heat flow (Site 858). These areas are linked by a "hydrothermal reservoir" (Site 857) of high-temperature fluids that has been well sealed by sediments. Site 856 is considered an area of former discharge where hydrothermal activity (possibly episodic) has led to strong alteration phenomena in the sedimentary cover and to formation of a large body of massive sulfides (Davis, Mottl, Fisher, et al., 1992). In most of these sites, hydrothermal alteration of the sediment consists of secondary precipitation of Mg-rich phyllosilicates and carbonate concretions. Even though the interpretation of the hydrologic environments of the drilled sites may be modified by postcruise results on fluid geochemistry, this paper accepts the Scientific Party's initial characterizations, while discussing the contributions

'Mottl, M.J., Davis, E.E., Fisher, A.T., and Slack, J.F. (Eds.), 1994. Proc. ODP, Sci. Results, 139: College Station, TX (Ocean Drilling Program).

${ }^{2}$ Université Lille 1, UFR des Sciences de la Terre, Lab. de Sédimentologie et Géodynamique Structural, F-59655 Villeneuve d'Ascq, France.

${ }^{3}$ CNRS, Centre de Géochimie de la Surface, 1 rue Blessig. F-67084 Strasbourg Cedex, France.

${ }^{4}$ Dip. di Scienze della Terra dell'Università di Napoli, Largo S. Marcellino 10, I-80138 Napoli, Italy.

${ }^{5}$ Dept. of Earth Sciences, ETH-Zïrich, CH-8092, Switzerland. resulting from our data to the resolution of the hydrologic problems of Middle Valley.

The aim of this study is to investigate the petrography, mineralogy, and geochemistry of the modifications induced by hydrothermal activity in the clastic sedimentary sequences of Middle Valley. Our investigations concern diagenetic low-temperature deposits and higher temperature products in the areas of maximum heat flow. Most of the analyses are focused on Sites 856 (relic discharge) and 858 (active discharge), where a more complete set of petrographic, mineralogic, and geochemical data was obtained. Only preliminary mineralogy and geochemistry are presented for Site 857 (hydrothermal reservoir).

In this paper, we report on the mineralogic and petrographic characteristics of both the detrital and authigenic mineral assemblages and discuss the hydrothermal alteration of the sedimentary cover at Middle Valley. Geochemical results, mainly from stable isotope geochemistry of the silicate and carbonate phases, are presented by Früh-Green et al. (this volume).

\section{GEOLOGIC SETTING}

Middle Valley is a fault-bounded, sedimented rift valley situated at the northern extremity of the Juan de Fuca Ridge, just south of the Sovanco fracture zone (Fig. 1). Its geologic setting has been described by Davis et al. (1987), Davis and Villinger (1992), and Davis, Mottl, Fisher, et al., (1992). Because of its proximity to the North American continental margin, Middle Valley is principally filled by terrigenous sediments (Goodfellow and Blaise, 1988) consisting of turbidite units of variable thickness, irregularly interbedded with biogenic pelagic sediments. The thickness of the sedimentary sequence (about $100 \mathrm{~m}$ to probably over $2000 \mathrm{~m}$ ) generally increases from the margins to the center of the basin, and from south to north toward the intersection of the rift with the Sovanco transform fault zone (Davis and Lister, 1977). During a period of maximum sedimentation in the Pleistocene, sedi- 


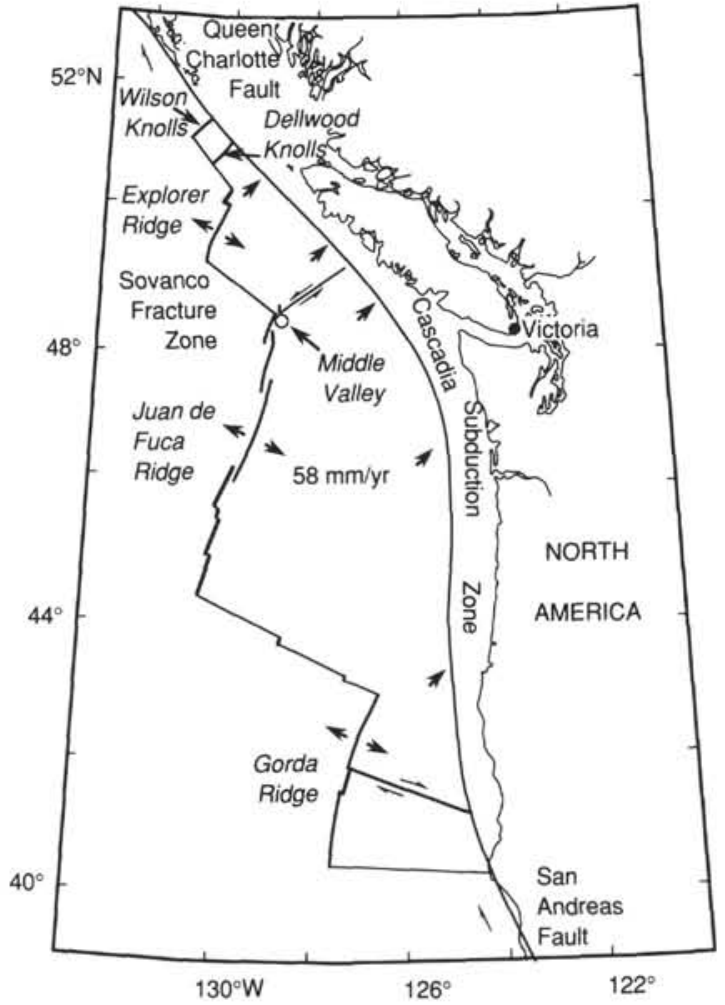

Figure 1. Location map showing the tectonic setting of Leg 139 in Middle Valley of the Juan de Fuca Ridge (from Davis, Mottl, Fisher, et al., 1992).

ment supply from the continental margin was high because there were no barriers blocking turbidite supply to the valley. After the Pleistocene, the tectonic regime gradually changed at the location of the drill sites. Uplift of the fault blocks into which the holes were drilled caused accumulation rates to decrease in the latest Pleistocene (Brunner, this volume). In the Holocene, the sediment supply from the continental margin, as well as deep-sea sedimentation, have been quite low (Al-Aasm and Blaise, 1991). The elevated basement temperatures estimated in the valley (Davis and Villinger, 1992) probably reflect the high thermal insulating capacity and low permeability of the sedimentary cover, which play an important role in maintaining the high temperature of fluids and in focusing hydrothermal discharge at a restricted number of vent sites (Goodfellow and Blaise, 1988). Numerous local heat flow anomalies occur in the valley (Fig. 2). Sites 856 and 858 are known to be associated with the discharge of hydrothermal fluids at the seafloor at temperatures similar to those estimated for the top of the permeable basement in the surrounding region.

\section{Site 856: Former Hydrothermal Discharge Site}

Site 856 is located around a small circular hill (Bent Hill) in the eastern part of Middle Valley, about $3 \mathrm{~km}$ west of the fault scarp forming the eastern topographic boundary of the valley (Fig. 2). There is no heat-flow anomaly directly associated with the hill and it appears to be thermally extinct (Davis and Villinger, 1992). Eight holes were drilled in a concentrated coverage on and around Bent Hill; six of them, $856 \mathrm{C}, 856 \mathrm{D}, 856 \mathrm{E}, 856 \mathrm{~F}, 856 \mathrm{G}$ and $856 \mathrm{H}$, encountered abundant massive sulfides. The other two holes, $856 \mathrm{~A}$ on the top of Bent Hill, and 856B to the south, recorded strong evidence for hydrothermal alteration within the clastic sediments. Holes $856 \mathrm{~A}$ and 856B could be considered the more distal portion of an asymmetric fossil vent field. Our mineralogic and petrographic study focuses on Holes $856 \mathrm{~A}$ and $856 \mathrm{~B}$ at this site.

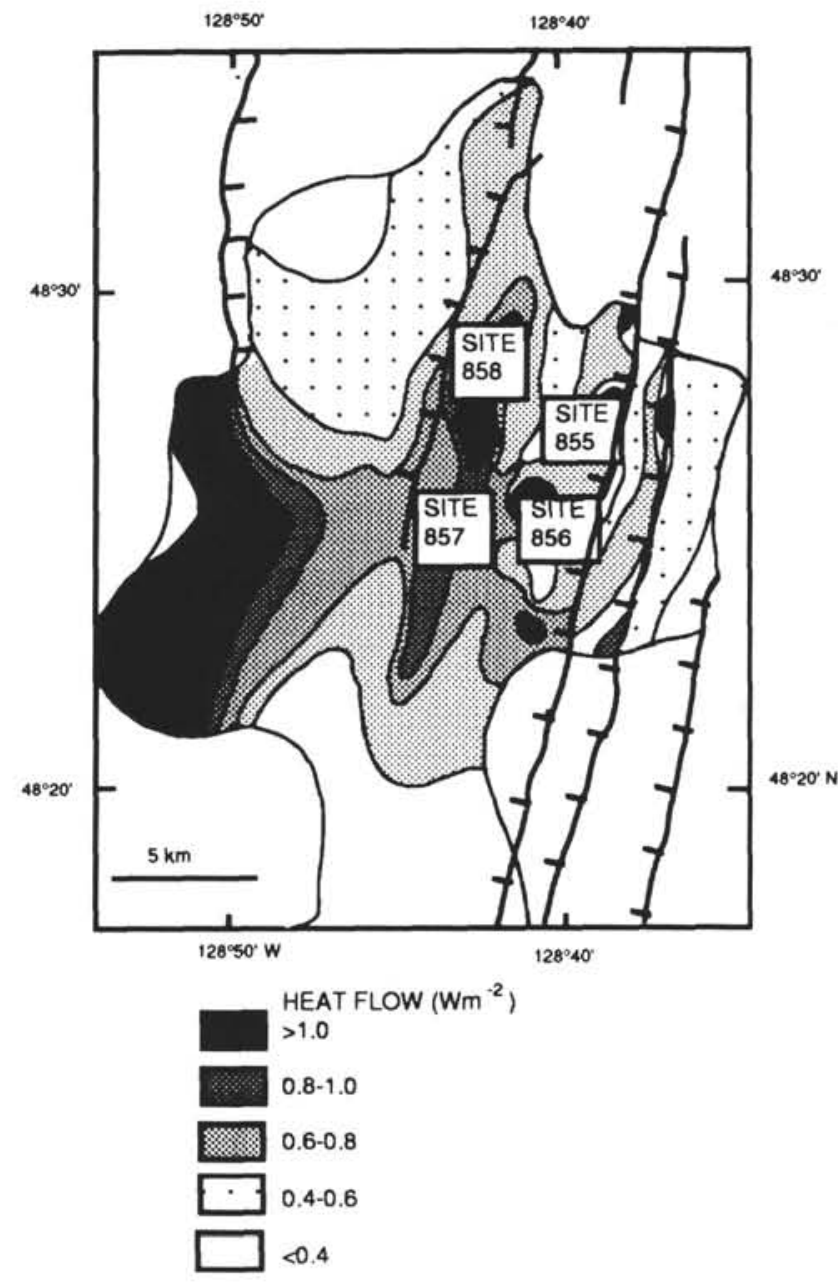

Figure 2. Heat flow in the southern part of Middle Valley (after Davis, Mottl, Fisher, et al., 1992).

\section{Site 857: Hydrothermal Reservoir Site}

Site 857 is located $5.2 \mathrm{~km}$ west of the fault scarp of the valley and about $1.5 \mathrm{~km}$ east of the fault that forms the current structural boundary of the central rift (Fig. 2). It is $1.6 \mathrm{~km}$ south of an active vent area (Site 858). The site is situated on a buried basement fault block standing 1 $\mathrm{km}$ above the basement level in the center of the valley. Although no discharge activity has been recorded here, the site is located over a linear thermal anomaly that parallels the rift valley about $1 \mathrm{~km}$ east of the primary rift-bounding fault (Davis, Mottl, Fisher, et al., 1992). At Site 857 four holes were cored, at distances ranging from a few meters to $180 \mathrm{~m}$ between each hole. Holes $857 \mathrm{~A}$ and $857 \mathrm{C}$ have been studied here.

\section{Site 858: Active Hydrothermal Discharge Site}

Site 858 is located about $6 \mathrm{~km}$ east of the present-day axis of the rift valley, in the middle of an active 800-m-long and 400-m-wide vent area. The vent field is situated over a local basement high, and the sediment thickness in the area ranges from 400 to $700 \mathrm{~m}$ below seafloor. Fluids discharge through numerous scattered vents at temperatures between $255^{\circ}$ and $275^{\circ} \mathrm{C}$. Heat flow in the area surrounding the vent field decreases systematically with distance from the field, falling to background levels over a distance of a few hundred meters (Davis and Villinger, 1992). Of the five holes that were drilled across the heat-flow anomalies and that penetrated the sedimentary piles, 
Hole $858 \mathrm{~A}$ is $100 \mathrm{~m}$ west of the vent field area, Hole $858 \mathrm{C}$ is about $70 \mathrm{~m}$ west of the nearest active vent (within the distal part of the vent field), Hole $858 \mathrm{~B}$ is only a few meters away from an active hydrothermal vent, and Holes $858 \mathrm{D}$ and $858 \mathrm{~F}$ are in the center of the venting area, about $70 \mathrm{~m}$ northeast of the vent drilled at Hole 858B. Data on Holes $858 \mathrm{~A}, 858 \mathrm{~B}, 858 \mathrm{C}$, and $858 \mathrm{D}$ are presented in this study.

\section{SEDIMENTARY SEQUENCES}

During Leg 139, sediments recovered from Middle Valley were divided into several units that can be correlated from one hole to another (Davis, Mottl, Fisher, et al., 1992). Criteria for division are the amount of turbidites in the sedimentary sequence and the degree of alteration. The succession of the lithologic units for each site is presented in Figure 3.

Unit I, which was recovered in the shallowest part of most of the cores, consists of hemipelagic sediments of Holocene age, with minor turbidite and abundant biogenic components. At Site 858, biogenic components decrease in holes closer to the venting areas and are absent in Holes 858B, 858C, and 858D below 13 mbsf.

Unit II, which consists of interbedded hemipelagic and turbiditic sediments, is subdivided into several subunits: Subunit IIA consists of weakly altered and indurated sediments, with abundant carbonate and/or dolomite nodules and concretions. Sediments of Subunit IIB are more strongly altered than those of Subunit IIA; the sediments are partially indurated and brecciated, and carbonate nodules, concretions, and fracture fillings are common. Subunit IIC is similar to Subunit IIB but shows more alteration, fracturing, and brecciation. The sediments of Subunit IID are strongly indurated and brecciated; at Site 858 they are silicified.

Unit III, which is present only in Hole $858 \mathrm{~B}$, is interbedded with Unit I and composed of metalliferous, oxidized, smectite-rich muds. Unit IV, recovered only at Sites 858 and 856 , consists of massive sulfide deposits. Unit V, present in Holes $858 \mathrm{~F}$ and $858 \mathrm{G}$, is made up of the igneous basement, and Unit VI consists of mafic intrusions.

It should be noted that the units defined by the shipboard party during Leg 139 are based on lithologic character and do not necessarily reflect a stratigraphic sequence of deposition. This is particularly evident in Hole 858B, where Units II and IV represent metalliferous intervals within hemipelagic and turbiditic sediments (see Davis, Mottl, Fisher, et al., 1992).

\section{METHODS}

Samples analyzed in this study are from Sites 856,857 , and 858. The clay-fraction study was focused on Site 858 (Table 1).

Routine X-ray diffraction (XRD) analyses were made on bulk powdered samples and on individual fragments using a Philips PW1710 diffractometer. Samples were run between $3^{\circ}$ and $65^{\circ} 2 \theta 40$ $\mathrm{kV} / 20 \mathrm{~mA}$, using $\mathrm{CuK} \alpha$ radiation, a Ni filter, and a scan speed of $1 \% \mathrm{~min}$. Quantitative XRD analysis is difficult in polyphased heterogeneous mixtures (Eberhart, 1989), and the relative abundances of minerals were estimated from the height of the main reflection and comparisons with the reflections of other samples. Qualitative data are given in Tables 2, 3, and 4. Scanning electron microscope (SEM) observations were made on Au-coated fragments of bulk samples using a JEOL JSM840 scanning electron microscope operated at $80 \mathrm{kV}$.

Petrographic analysis of about 50 thin sections of carbonate-rich samples was complemented by cathodoluminescence microscopic observation, performed on polished thin sections and slabs, using a Technosyn 8200 MkIII cold cathode with a beam voltage of $15 \mathrm{kV}$ and a current of $200 \mu \mathrm{A}$. Photographs were taken under a current of $550 \mu \mathrm{A}$. The electron microprobe analysis of carbonate concretions was performed on a five-spectrometer CAMECA SX 50, using both wavelength-dispersive and energy-dispersive modes. The accelerating voltage was $15 \mathrm{kV}$, with a beam current of about $15 \mathrm{nA}$. Wollastonite $(\mathrm{Ca})$, magnetite $(\mathrm{Fe})$, periclase $(\mathrm{Mg})$, celsian $(\mathrm{Ba})$, and celestine

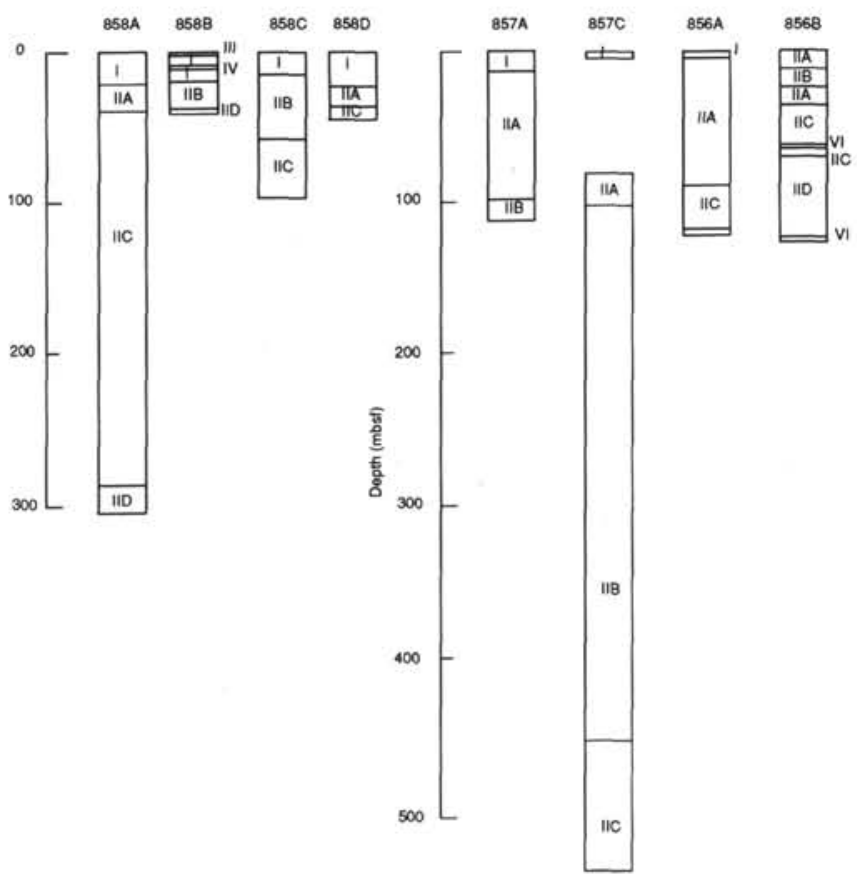

Figure 3. Summary of the lithostratigraphic units for the studied holes at Sites 856,857 , and 858 .

( $\mathrm{Sr}$ ) were used as standards for these elements, and pure metals were used for $\mathrm{Mn}, \mathrm{Cu}$, and $\mathrm{Zn}$.

In preparation for XRD analyses of the clay fraction, each sample was dispersed in deionized water, disaggregated, decarbonated with an $\mathrm{HCl} \mathrm{N} / 5$ solution, and washed several times before separation of the $<2-\mu \mathrm{m}$ fraction by settling in a water column. A preparation of oriented aggregates of clays was then collected on a glass slide. The samples were sequentially air-dried, glycolated, and heated for $4 \mathrm{hrs}$ at $490^{\circ} \mathrm{C}$, for identification of interlayering of clays, i.e., the (001) basal reflection positions after each treatment. The analyses were performed with a Philips X-ray diffractometer utilizing a scintillation detector, graphite monochromator, $\mathrm{CuK} \alpha$ radiation, and theta-compensating slits. The diffractometer was controlled by a microcomputer. The XRD patterns were obtained between $2^{\circ}$ to $32^{\circ} 2 \theta$. Analyses were also made on random powder samples between $59^{\circ}$ to $64^{\circ} 2 \theta$ for the study of $(060)$ reflections. The relative abundances of clay minerals reported in Tables 6 and 7 were estimated using the same methods as those described for the bulk samples. Smectite was detected by the (001) reflection at 14.8 $\AA$ and the (002) reflection at $8.5 \AA$; after glycolation, the (001) reflection shifts to $17 \AA$. Corrensite, a smectite-chlorite in regularly mixed layers (R1), shows (001) reflections at $31 \AA, 14.9 \AA, 9.9 \AA, 7.4 \AA, 5.9$ $\AA, 4.9 \AA, 3.6 \AA, 3.3 \AA$, and $2.9 \AA$. After glycolation, the first three reflections shift to $32.9 \AA, 16 \AA$, and $7.9 \AA$. Swelling chlorite is a chlorite with expandable layers or chlorite/smectite random mixed layers: the (001) reflections are at $14.2 \AA(001)$ and $7.1 \AA(002)$ and expand slightly after glycolation to $14.7 \AA$ and $7.4 \AA$, indicating a very high proportion $(>70 \%)$ of chlorite layers. The peaks are broad in comparison to the chlorite reflections. Chlorite was detected by reflections $(001)$ at $14.2 \AA,(002)$ at $7.1 \AA,(003)$ at $4.75 \AA$, and $(004)$ at 3.55 $\AA$. The reflection positions are the same after glycolation and heating. The low intensity of odd reflections in comparison with even reflections in the spectra of chlorite-rich samples (Fig. 4) reflect the high $\mathrm{Fe}$-content of the chlorite.

Chemical analyses were run on the $<2-\mu \mathrm{m}$ fraction of a few samples. Prior to elemental analysis, the clay fractions were dried at $110^{\circ} \mathrm{C}$, melted in a mixture of lithium tetraborate, and introduced into a glycolated solvent. Major element analyses were performed following the method described by Besnus and Rouault (1973) using arc spectrome- 


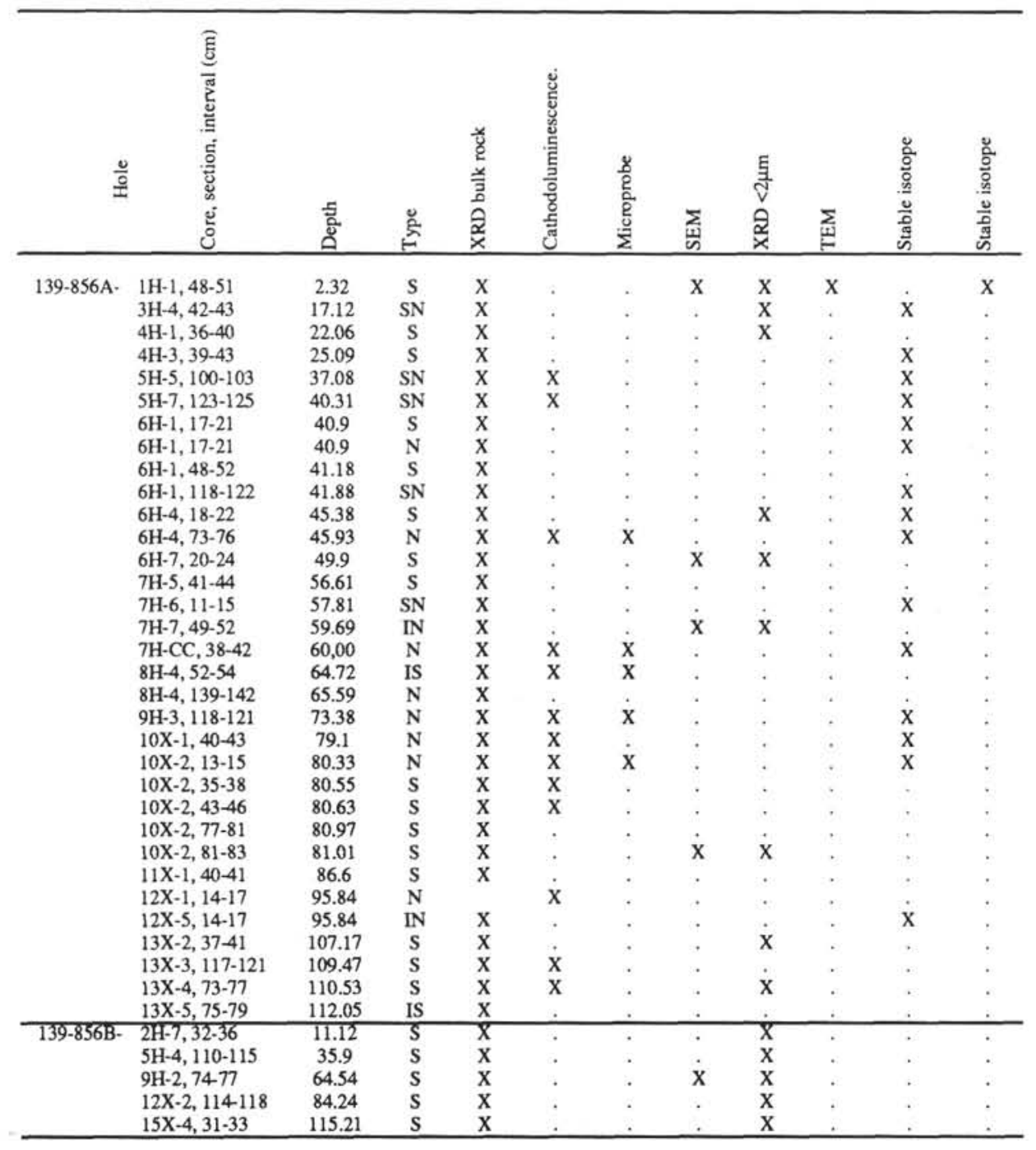

Note: type of samples: $\mathrm{S}=$ soft mud, $\mathrm{SN}=$ soft nodule, $\mathrm{IN}=$ indurated nodule, $\mathrm{N}=$ nodule,

$\mathrm{BR}=$ breccia, $\mathrm{MA}=$ matrix, $\mathrm{IS}=$ indurated mud, $\mathrm{MS}=$ metamorphic mud.

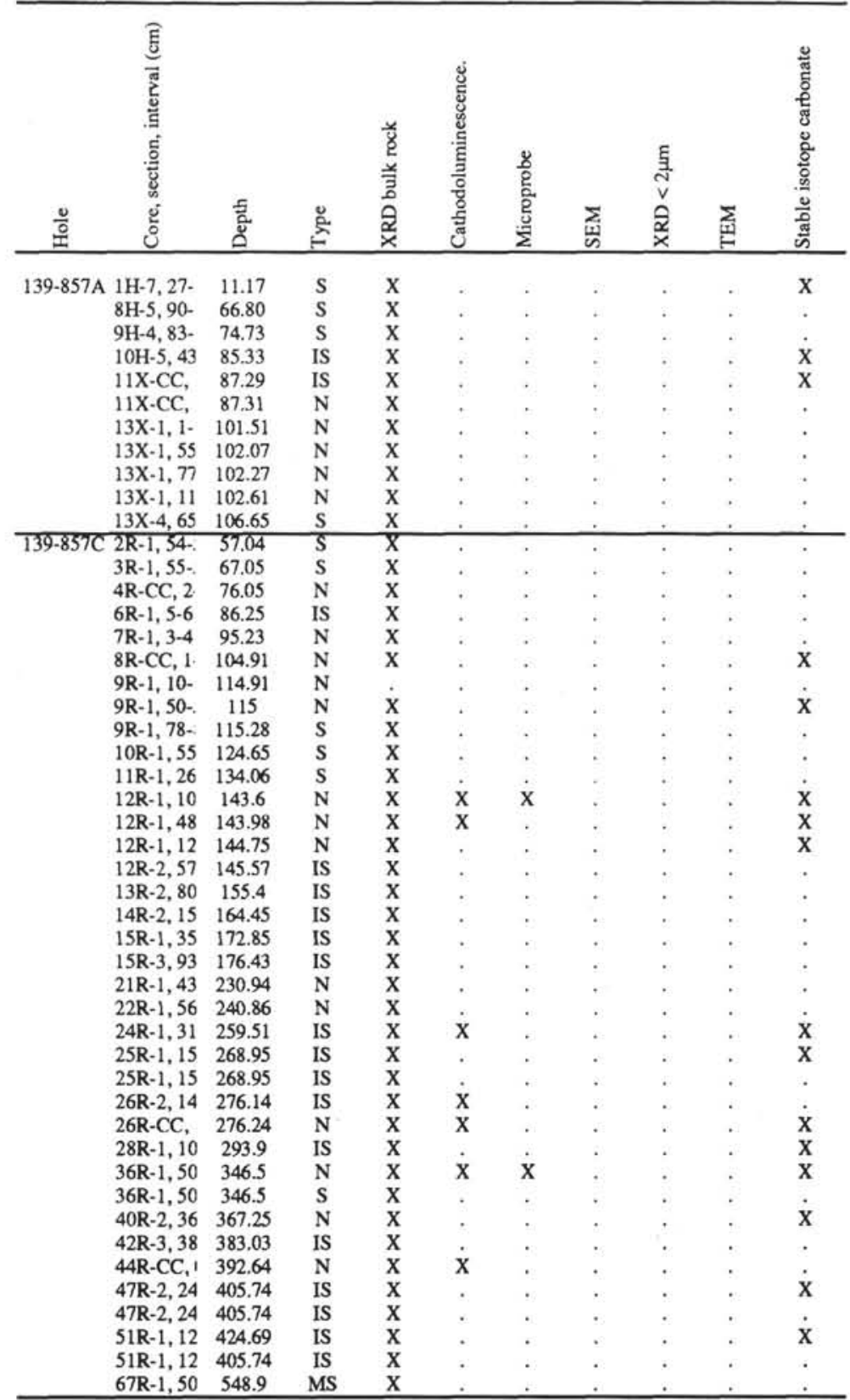


Table 1 (continued).

\begin{tabular}{|c|c|c|c|c|c|c|c|c|c|c|c|}
\hline & 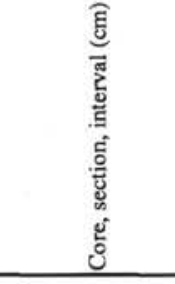 & 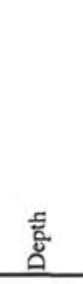 & 总 & 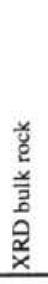 & 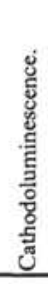 & 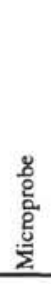 & 䍃 & 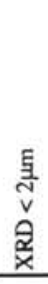 & $\underset{E}{\sum}$ & 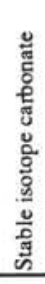 & 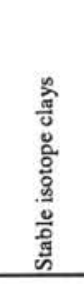 \\
\hline \multirow[t]{20}{*}{$139-858 \mathrm{~A}-$} & $2 \mathrm{H}-3,36-40$ & 5.76 & $s$ & $\mathrm{x}$ & . & . & $\mathrm{x}$ & $\mathrm{x}$ & . & & $\mathrm{x}$ \\
\hline & $2 \mathrm{H}-7,30-31$ & 11.7 & $\mathrm{~N}$ & $\mathrm{x}$ & . & . & . & . & . & $\mathrm{x}$ & . \\
\hline & $2 \mathrm{H}-7,38-40$ & 11.78 & $\mathrm{~N}$ & $\mathrm{x}$ & . & . & . & . & . & & . \\
\hline & $2 \mathrm{H}-7,40-42$ & 11.8 & $\mathrm{~N}$ & $\mathrm{x}$ & $\mathrm{x}$ & . & . & . & . & $\mathrm{x}$ & . \\
\hline & $3 \mathrm{H}-1,0-3$ & 11.9 & $\mathrm{~N}$ & $\mathrm{x}$ & $\mathrm{x}$ & $\mathrm{x}$ & . & & . & $\mathrm{x}$ & . \\
\hline & $3 \mathrm{H}-1,58-60$ & 12.48 & $s$ & $\mathrm{x}$ & & . & . & $\mathrm{x}$ & . & & . \\
\hline & $4 \mathrm{H}-7,15-17$ & 30.55 & S & $\mathrm{x}$ & $\mathrm{x}$ & . & . & $\mathrm{x}$ & . & $\mathrm{x}$ & . \\
\hline & $5 \mathrm{H}-2,2-4$ & 32.42 & $\mathrm{~N}$ & & & . & . & . & . & . & . \\
\hline & $5 \mathrm{H}-2,134-137$ & 33.74 & $\mathrm{~N}$ & $\mathrm{x}$ & $\mathrm{x}$ & . & . & . & . & . & . \\
\hline & $5 \mathrm{H}-4,27-30$ & 35.67 & $\mathrm{~S}$ & $\mathrm{x}$ & & & $\mathrm{x}$ & $\mathrm{x}$ & $\mathrm{x}$ & . & $\mathrm{x}$ \\
\hline & $5 \mathrm{H}-6,40-45$ & 38.8 & $\mathrm{~N}$ & & $\mathrm{x}$ & $\mathrm{x}$ & . & . & . & . & . \\
\hline & $5 \mathrm{H}-7,10-11$ & 40,00 & $s$ & . & $\mathrm{x}$ & $\mathrm{x}$ & . & . & . & . & . \\
\hline & $8 \mathrm{H}-2,57-59$ & 60.9 & $\mathrm{~N}$ & $x$ & $\mathrm{x}$ & . & . & . & . & $\mathrm{x}$ & . \\
\hline & $9 \mathrm{X}-2,61-64$ & 64.61 & $\mathrm{~N}$ & & $\mathrm{x}$ & . & . & . & . & $\mathrm{x}$ & . \\
\hline & $11 \mathrm{X}-\mathrm{CC}, 12-14$ & 73.59 & $s$ & $x$ & . & . & $\therefore$ & $x$ & . & . & . \\
\hline & $12 \mathrm{X}-\mathrm{CC}, 14-15$ & 81.94 & $s$ & $\mathrm{x}$ & . & . & $\mathrm{x}$ & $\mathrm{x}$ & . & & $\mathrm{x}$ \\
\hline & $14 \mathrm{X}-\mathrm{CC}, 11-13$ & 101.11 & $\mathrm{~N}$ & $\mathrm{x}$ & . & . & . & $\mathrm{x}$ & . & $\mathrm{x}$ & \\
\hline & $18 \mathrm{X}-2,132-134$ & 142.42 & $s$ & $\mathrm{x}$ & . & . & . & $\mathrm{x}$ & . & & $\mathrm{x}$ \\
\hline & $27 \mathrm{X}-1,24-26$ & 226.97 & $S$ & $\mathrm{x}$ & . & . & $\mathrm{x}$ & $\mathrm{x}$ & . & . & 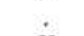 \\
\hline & $31 X-2,3-5$ & 266.17 & $s$ & $\mathrm{x}$ & . & . & $\mathrm{x}$ & $\mathrm{x}$ & . & & $\mathrm{x}$ \\
\hline \multirow{20}{*}{$139-858 \mathrm{~B}-$} & $1 H-1,124-126$ & 1.24 & $S$ & & . & . & . & ? & . & & . \\
\hline & $1 \mathrm{H}-2,129-133$ & 2.79 & S & $\mathrm{x}$ & . & & . & . & . & $\mathrm{x}$ & . \\
\hline & $1 \mathrm{H}-4,108-112$ & 5.58 & $\mathrm{~s}$ & $\mathrm{x}$ & . & . & . & . & . & & . \\
\hline & $1 \mathrm{H}-5,34-36$ & 6.34 & $\mathrm{~s}$ & $x$ & . & . & . & . & . & $\mathrm{x}$ & . \\
\hline & $2 \mathrm{H}-1,97-99$ & 8.17 & s & $\mathrm{X}$ & . & . & $\mathrm{x}$ & $\mathrm{x}$ & & & $\mathrm{x}$ \\
\hline & $2 \mathrm{H}-2,45-47$ & 9.15 & s & $\mathrm{x}$ & . & . & & $\mathrm{x}$ & $\mathrm{x}$ & . & $\mathrm{x}$ \\
\hline & $2 \mathrm{H}-3,75-77$ & 10.95 & $\mathrm{~s}$ & $\mathrm{x}$ & . & . & $\mathrm{x}$ & $\mathrm{x}$ & $\mathrm{x}$ & . & $\mathrm{x}$ \\
\hline & $2 \mathrm{H}-3,126-129$ & 11.45 & $S-B R$ & $\mathrm{x}$ & $\mathrm{x}$ & . & . & . & . & . & . \\
\hline & $2 \mathrm{H}-3,126-129$ & 11.45 & S-MA & $\mathrm{x}$ & & . & . & . & . & . & \\
\hline & $2 \mathrm{H}-4,72-74$ & 12.42 & $\mathrm{~s}$ & $\mathrm{x}$ & . & . & $\mathrm{x}$ & $x$ & . & & $\mathrm{x}$ \\
\hline & $2 \mathrm{H}-5,113-115$ & 14.33 & s & $\mathrm{x}$ & . & . & . & $\mathrm{x}$ & . & . & $\mathrm{x}$ \\
\hline & $2 \mathrm{H}-6,89-91$ & 15.59 & $s$ & $\mathrm{x}$ & . & . & 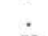 & $\mathrm{x}$ & . & . & $\mathrm{x}$ \\
\hline & $5 \mathrm{H}-2,69-73$ & 26.09 & s & $\mathrm{x}$ & . & . & $\mathrm{x}$ & $\mathrm{x}$ & . & & \\
\hline & $5 \mathrm{H}-3,58-62$ & 27.48 & s & $\mathrm{x}$ & . & . & $\mathrm{x}$ & $\mathrm{x}$ & . & . & $\mathrm{X}+\mathrm{XQ}$ \\
\hline & $5 \mathrm{H}-4,55-59$ & 28.95 & $s$ & $\mathrm{x}$ & . & . & $\mathrm{x}$ & $\mathrm{x}$ & $\mathrm{x}$ & . & $\mathrm{x}$ \\
\hline & $6 \mathrm{H}-1,51-53$ & 32.01 & $s$ & $\mathrm{x}$ & & . & $\mathrm{x}$ & $\mathrm{x}$ & $\mathrm{x}$ & . & $\mathrm{X}+\mathrm{XQ}$ \\
\hline & $6 \mathrm{H}-1,67-70$ & 32.15 & IS & $\mathrm{x}$ & $\mathrm{x}$ & . & . & . & . & . & . \\
\hline & $8 \mathrm{X}-1,27-31$ & 32.97 & IS & . & . & . & . & . & . & . & . \\
\hline & $8 \mathrm{X}-2,26-28$ & 34,00 & IS & . & . & . & $\mathrm{x}$ & $\mathrm{x}$ & . & , & . \\
\hline & $8 X-4,22-24$ & 36.3 & IS & & & . & $\mathrm{x}$ & $\mathrm{x}$ & . & $\therefore$ & . \\
\hline
\end{tabular}

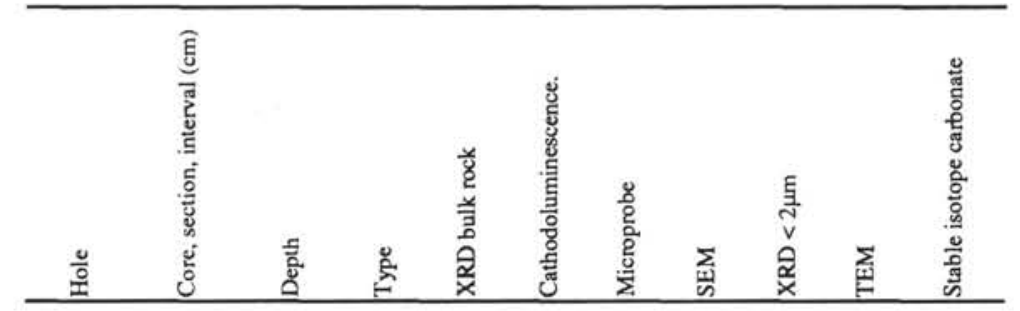

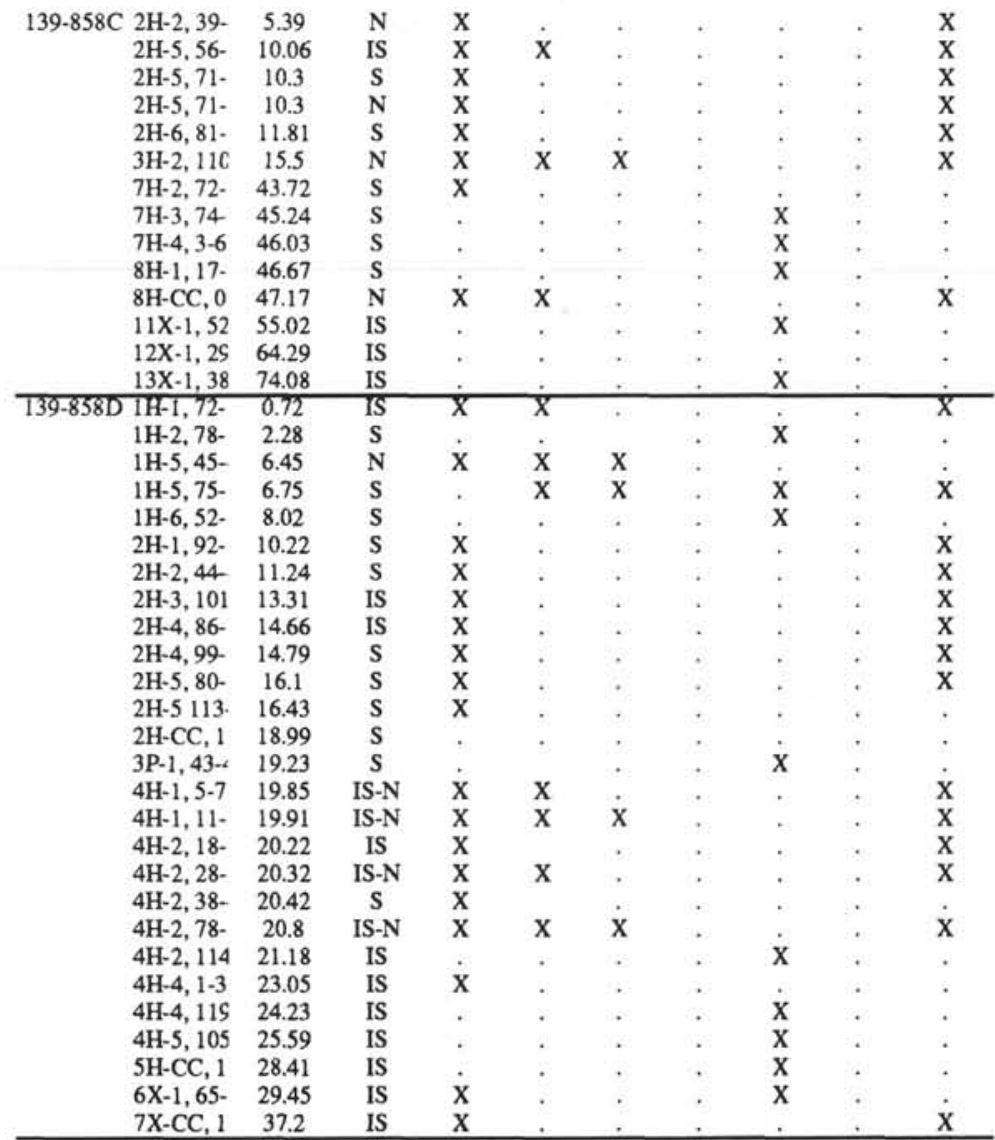


try and an ARL quantimeter. Na and $\mathrm{K}$ contents were determined by emission spectrometry. Major elements are expressed in percentage of oxides, and the weight loss on ignition ( $\mathrm{LOI}$, at $1000^{\circ} \mathrm{C}$ ) in percentage for $100 \mathrm{~g}$ of dried samples with a relative precision of $\pm 2 \%$.

Clay suspensions were settled on a carbon copper grid for particle morphology observation with a Philips CM30 transmission electron microscope (TEM) operated at $300 \mathrm{kV}$.

\section{RESULTS}

\section{Bulk-Sediment Mineralogy}

XRD investigation of the bulk sediments was conducted to identify the mineral assemblages of the deposits and associated carbonate concretions, and to determine the various origins of the sediment components (Tables 2 through 4). The nature of the deposits, as well as the relations among the phases, were investigated by SEM on bulkrock fragments.

Quartz, feldspar, mica, and amphibole are considered detrital in origin, representing the background hemipelagic sedimentary supply (Davis, Mottl, Fisher, et al., 1992). Nevertheless, SEM observations demonstrate that quartz is locally authigenic. Hydrothermal input is well documented by the presence of sulfate and sulfide concentrations. The carbonates are mainly calcite and dolomite, forming primary or secondary hydrothermally modified phases. Clay minerals, of both detrital and authigenic origin, are described in more detail in the "Clay Mineral Assemblages" section, this chapter.

\section{Former Hydrothermal Discharge Site 856}

The proportions of quartz, feldspar, and mica are unvarying in all units throughout the sedimentary sequence at Holes $856 \mathrm{~A}$ and $856 \mathrm{~B}$, regardless of grain size or induration (Table 2). Minor amphiboles occur in the uppermost units. In the surficial sediments forming Unit I, the detrital assemblage is mixed with biogenic pelagic phases, such as large radiolarian tests and diatom fragments (Pl. 1, Fig. 1). SEM observations of this unit confirm the detrital origin of the quartz, feldspars, and micas, evidenced by rounded grain edges and traces of dissolution. In deeper deposits, corroded quartz and feldspar grains with rounded edges are still observed (Pl. 1, Fig. 2). A few of the samples contain minor pyrite, commonly expressed as framboidal grains and concretions. Clay minerals are abundant in the sediments, and become prevalent in the more hydrothermally modified sediments at Hole 856B. Carbonate concretions concentrated in the turbiditic beds (Hole 856A, Subunit IIA) are composed of calcite with low $\mathrm{Mg}$ substitution (Früh-Green et al., this volume). The relative abundance of the clay phases and carbonates is negatively correlated, both within indurated sediments and concretions. This negative correlation seems to be the main expression of the hydrothermal alteration of the clastic sediments.

\section{Hydrothermal Reservoir Site 857}

Sediments drilled at Holes $857 \mathrm{~A}$ and $857 \mathrm{C}$ in which induration increases with depth appear free of significant hydrothermal sulfate

Table 2. Bulk mineralogic composition of the sediments and nodules from Site 856 estimated by X-ray diffraction analyses on powdered samples.

\begin{tabular}{|c|c|c|c|c|c|c|c|c|c|c|c|}
\hline & $\begin{array}{l}\text { Core, section } \\
\text { interval }(\mathrm{cm})\end{array}$ & $\begin{array}{l}\text { Depth } \\
\text { (mbsf) }\end{array}$ & Unit & Type & Quartz & $\begin{array}{l}\text { feldspar+ } \\
\text { Amphibole }\end{array}$ & Mica & Clays & Calcite & Sulfates & Pyrite \\
\hline \multicolumn{12}{|l|}{$139.856 \mathrm{~A}-$} \\
\hline & $3 \mathrm{H}-4,42-43$ & $\begin{array}{r}2,32 \\
17,12\end{array}$ & IIA & SN & $\hat{x}$ & $\hat{\mathrm{o}}$ & o & $\hat{X}$ & $X X$ & . & : \\
\hline & $4 \mathrm{H}-1,36-40$ & 22,06 & IIA & $\mathrm{S}$ & $\mathrm{XX}$ & O & $\mathrm{X}$ & $\mathrm{XX}$ & 0 & . & . \\
\hline & $4 \mathrm{H}-3,39-43$ & 25,09 & IIA & $\mathrm{S}$ & $X X$ & $\mathrm{X}$ & $\mathrm{X}$ & $\mathrm{XX}$ & $\mathrm{O}$ & , & . \\
\hline & $5 \mathrm{H}-5,100-103$ & 37,08 & IIA & SN & $X X$ & $\mathrm{x}$ & $\mathrm{x}$ & $\mathrm{X}$ & $\mathrm{x}$ & . & . \\
\hline & $6 \mathrm{H}-1,17-21$ & 40,87 & IIA & $\mathrm{N}$ & $X X$ & $\mathrm{X}$ & . & 0 & $\mathrm{xx}$ & . & . \\
\hline & $6 \mathrm{H}-1,48-52$ & 41,18 & IIA & $\mathrm{S}$ & $\mathrm{XX}$ & $\mathrm{x}$ & $\mathrm{X}$ & $\mathrm{XX}$ & $\cdot$ & . & . \\
\hline & $6 \mathrm{H}-1,118-122$ & 41,88 & IIA & SN & $\mathrm{XX}$ & $\mathrm{X}$ & $\mathrm{XX}$ & $\mathrm{X}$ & 0 & . & . \\
\hline & $6 \mathrm{H}-4,18-22$ & 45,38 & IIA & $\mathrm{S}$ & $\mathrm{XX}$ & $\mathrm{X}$ & 0 & $\mathrm{XX}$ & $\mathrm{x}$ & . & . \\
\hline & $6 \mathrm{H}-4,73-76$ & 45,93 & IIA & $\mathrm{N}$ & $\mathrm{XX}$ & $\mathrm{x}$ & . & 0 & $\mathrm{XX}$ & . & . \\
\hline & $6 \mathrm{H}-7,20-24$ & 49,90 & IIA & $\mathbf{S}$ & $\mathrm{XX}$ & $\mathrm{X}$ & $\mathrm{X}$ & $\mathrm{XX}$ & $\mathrm{X}$ & . & . \\
\hline & $7 C C, 38-42$ & 59,70 & IIA & $\mathrm{N}$ & $x x$ & $\mathrm{x}$ & . & $\mathrm{O}$ & $\mathrm{XX}$ & , & . \\
\hline & $8 \mathrm{H}-4,52-54$ & 64,72 & IIA & IS & $\mathrm{Xx}$ & $\mathrm{x}$ & $\mathrm{x}$ & $\mathrm{XX}$ & . & . & . \\
\hline & $8 \mathrm{H}-4,139-142$ & 65,59 & IIA & $\mathrm{N}$ & $\mathrm{XX}$ & $\mathrm{XX}$ & . & $\cdot$ & $\cdot$ & . & $\mathrm{XX}$ \\
\hline & $9 \mathrm{H}-3,118-12 \mathrm{I}$ & 73,38 & IIA & $\mathrm{N}$ & $\mathrm{XX}$ & $\mathrm{x}$ & . & O & $\mathrm{XXX}$ & . & • \\
\hline & $10 X-1,40-43$ & 79,10 & IIA & $\mathrm{N}$ & $\mathrm{XX}$ & $\mathrm{x}$ & . & O & $\mathrm{XXX}$ & . & . \\
\hline & $10 X-2,13-15$ & 80,33 & IIA & $\mathbf{N}$ & $x x$ & $\mathrm{XX}$ & . & $\mathrm{x}$ & $\mathrm{X}$ & . & . \\
\hline & $10 X-2,35-38$ & 80,55 & IIA & $\mathrm{S}$ & $\mathrm{XX}$ & $\mathrm{x}$ & $\mathrm{X}$ & $\mathrm{x}$ & $\mathrm{O}$ & . & . \\
\hline & $10 X-2,43-46$ & 80,63 & IIA & S & $\mathrm{XX}$ & $\mathrm{x}$ & $\mathrm{x}$ & $x x$ & $\cdot$ & . & , \\
\hline & $10 X-2,77-81$ & 80,97 & IIA & S & $\mathrm{XX}$ & $\mathrm{X}$ & $\mathrm{X}$ & $\mathrm{XX}$ & . & . & . \\
\hline & $10 X-2,81-83$ & 81,01 & ILA & $\mathbf{S}$ & $\mathrm{XX}$ & $\mathrm{X}$ & $\mathrm{X}$ & $\mathrm{X}$ & . & . & . \\
\hline & $12 X-5,14-17$ & 95,84 & IIC & IN & $\mathrm{xx}$ & $\mathrm{x}$ & . & $\mathrm{O}$ & $\mathrm{XX}$ & . & . \\
\hline & $13 X-2,37-41$ & 107,17 & IIC & $\mathrm{S}$ & $\mathrm{X}$ & $\mathrm{X}$ & $X$ & $\mathrm{XX}$ & • & . & - \\
\hline
\end{tabular}

Note : Type of sample: $\mathrm{S}=$ soft mud; $\mathrm{IS}=$ indurated mud; $\mathrm{N}=$ nodule: $\mathrm{SN}=\mathrm{Soft}$ nodule; $\mathrm{IN}=$ indurated nodule;

F. + Amp. $=$ Albite and amphiboles; Sulfates: $A=$ anhydrite, $G=$ gypsum; Clays: chlorite and smectite

Relative abundance: $\mathrm{X}=15 \%, \mathrm{O}=$ present, $\cdot=$ traces 
Table 3. Bulk mineralogic composition of the sediments and nodules from Site 857 estimated by $\mathrm{X}$-ray diffraction analyses on powdered samples.

\begin{tabular}{|c|c|c|c|c|c|c|c|c|c|c|}
\hline Hole & $\begin{array}{l}\text { Core, section } \\
\text { interval }(\mathrm{cm})\end{array}$ & $\begin{array}{l}\text { Depth } \\
\text { (mbsf) }\end{array}$ & Unit & Type & Quartz & Feldspar & Mica & Clays & Calcite & Dolomite \\
\hline \multicolumn{11}{|l|}{ 139-857A- } \\
\hline & $1 \mathrm{H}-7,27-31$ & 11,17 & I & $\mathbf{S}$ & $X X$ & $\mathbf{X}$ & $\mathrm{XX}$ & $\mathrm{X}$ & $\mathrm{O}$ & . \\
\hline & $8 \mathrm{H}-5,90-94$ & 66,80 & IIA & $\mathbf{S}$ & $\mathrm{XX}$ & $\mathrm{x}$ & $\mathbf{X}$ & $\mathrm{X}$ & O & . \\
\hline & $9 \mathrm{H}-4,83-88$ & 74,73 & IIA & $\mathbf{S}$ & $\mathrm{XX}$ & $\mathrm{XX}$ & $\mathrm{x}$ & $\mathrm{x}$ & o & . \\
\hline & $10 \mathrm{H}-5,43-45$ & 85,33 & IIA & IS & $\mathrm{XX}$ & $\mathrm{X}$ & O & 0 & . & $\mathrm{XX}$ \\
\hline & $11 \mathrm{X}-\mathrm{CC}, 22-24$ & 87,29 & IIA & IS & $\mathrm{XX}$ & $\mathrm{x}$ & $\mathrm{X}$ & $\mathrm{X}$ & . & $\mathrm{X}$ \\
\hline & $11 \mathrm{X}-\mathrm{CC}, 24-26$ & 87,31 & IIA & $\mathrm{N}$ & $\mathrm{X}$ & $\mathrm{x}$ & O & $\mathrm{x}$ & . & $\mathrm{XX}$ \\
\hline & $13 \mathrm{X}-1,1-2$ & 101,51 & IIB & $\mathrm{N}$ & $\mathrm{X}$ & o & . & . & . & $\mathrm{XXXX}$ \\
\hline & $13 X-1,55-57$ & 102,07 & IIB & $\mathrm{N}$ & $\mathrm{XX}$ & $\mathrm{x}$ & O & $\mathrm{XX}$ & . & . \\
\hline & $13 X-1,77-79$ & 102,27 & IIB & $\mathbf{N}$ & $\mathrm{XX}$ & $\mathrm{X}$ & 0 & $\mathrm{XX}$ & . & . \\
\hline & $13 X-1,111-114$ & 102,61 & IIB & $\mathbf{N}$ & $\mathrm{XX}$ & $\mathrm{X}$ & $\mathrm{X}$ & $\mathrm{X}$ & . & . \\
\hline & $13 X-4,65-67$ & 106,65 & IIB & IS & $\mathrm{XX}$ & $\mathrm{X}$ & $\mathrm{x}$ & $\mathrm{x}$ & . & . \\
\hline \multicolumn{11}{|l|}{$139.857 \mathrm{C}$. } \\
\hline & $2 \mathrm{R}-1,54-57$ & 57,04 & IIA & $\mathbf{S}$ & $\mathrm{XX}$ & $\mathrm{X}$ & $\mathrm{X}$ & $X$ & , & . \\
\hline & $3 R-1,55-57$ & 67,05 & IIA & $\mathrm{s}$ & $\mathrm{XX}$ & $\mathrm{X}$ & $\mathrm{x}$ & $\mathrm{XX}$ & . & . \\
\hline & $4 \mathrm{R}-\mathrm{CC}, 2-3$ & 76,12 & IIA & $\mathrm{N}$ & $\mathrm{X}$ & 0 & . & $\mathrm{X}$ & . & XXX \\
\hline & $6 R-1,5-6$ & 86,25 & IIA & IS & $\mathrm{x}$ & $\mathrm{x}$ & . & O & . & $\mathrm{XXX}$ \\
\hline & $7 \mathrm{R}-1,3-4$ & 95,23 & IIA & $\mathrm{N}$ & $\mathrm{XX}$ & $\mathrm{x}$ & . & 0 & . & $\mathrm{XXX}$ \\
\hline & $9 R-1,50-51$ & 115,00 & IIB & $\mathbf{N}$ & $\mathrm{XX}$ & $\mathrm{x}$ & . & O & $X X$ & · \\
\hline & $9 \mathrm{R}-1,78-80$ & 115,28 & IIB & $\mathbf{S}$ & $\mathrm{XX}$ & $\mathrm{x}$ & $\mathrm{x}$ & $\mathrm{X}$ & · & . \\
\hline & $10 \mathrm{R}-1,55-56$ & 124,65 & IIB & $\mathrm{S}$ & $\mathrm{XX}$ & $\mathrm{x}$ & $\mathrm{x}$ & $\mathrm{X}$ & . & . \\
\hline & $11 \mathrm{R}-1,26-28$ & 134,06 & IIB & $\mathbf{S}$ & $\mathrm{XX}$ & $\mathrm{x}$ & 0 & $\mathrm{XX}$ & $\therefore$ & . \\
\hline & $12 \mathrm{R}-1,10-11$ & 143,60 & IIB & $\mathbf{N}$ & $\mathrm{XX}$ & $\mathrm{x}$ & . & 0 & $\mathrm{XX}$ & . \\
\hline & $12 \mathrm{R}-1,48-49$ & 143,98 & IIB & $\mathrm{N}$ & $\mathrm{XX}$ & $\mathrm{x}$ & 0 & O & $\mathrm{XX}$ & . \\
\hline & $12 \mathrm{R}-1,125-126$ & 144,75 & IIB & $\mathrm{N}$ & $\mathrm{X}$ & $\mathrm{X}$ & . & 0 & $\mathrm{XXX}$ & . \\
\hline & $12 \mathrm{R}-2,57-60$ & 145,57 & IIB & IS & $\mathrm{XX}$ & $\mathrm{x}$ & 0 & $\mathrm{XX}$ & . & . \\
\hline & $13 R-2,80-84$ & 155,40 & IIB & IS & $\mathrm{X}$ & 0 & $\mathrm{XX}$ & $\mathrm{XX}$ & . & . \\
\hline & $14 \mathrm{R}-2,15-19$ & 164,45 & IIB & IS & $\mathrm{XX}$ & $\mathrm{XX}$ & 0 & $\mathrm{X}$ & . & . \\
\hline & $15 \mathrm{R}-1,35-37$ & 172,85 & IIB & IS & $\mathrm{XX}$ & $\mathrm{XX}$ & 0 & $\mathrm{XX}$ & . & . \\
\hline & $15 R-3,93-95$ & 176,43 & IIB & IS & $\mathrm{XX}$ & $\mathrm{X}$ & $\mathrm{X}$ & $\mathrm{XX}$ & . & . \\
\hline & $21 R-1,43-63$ & 230,94 & IIB & $\mathrm{N}$ & $\mathrm{XX}$ & $\mathrm{XX}$ & $\mathrm{X}$ & $\mathrm{X}$ & O & . \\
\hline & $22 R-1,56-59$ & 240,86 & IIB & $\mathrm{N}$ & $X$ & $X$ & $\mathrm{XX}$ & $X X$ & - & . \\
\hline & $24 \mathrm{R}-1,31-35$ & 259,51 & IIB & IS & $\mathrm{XX}$ & $\mathrm{X}$ & $\mathrm{X}$ & $\mathrm{XX}$ & O & . \\
\hline & $25 \mathrm{R}-1,15-17$ & 268,95 & IIB & IS & $X$ & $\mathrm{X}$ & $\mathrm{X}$ & $\mathrm{XX}$ & $\therefore$ & . \\
\hline & $25 \mathrm{R}-1,15-17$ & 268,95 & IIB & IS & $\mathrm{X}$ & $\mathrm{X}$ & o & $\mathrm{X}$ & $\mathrm{XX}$ & \\
\hline & $26 \mathrm{R}-2,14-16$ & 276,14 & IIB & IS & $\mathrm{XX}$ & $\mathrm{XX}$ & O & $\mathrm{X}$ & 0 & . \\
\hline & $26 \mathrm{R}-\mathrm{CC}, 1-3$ & 276,24 & IIB & $\mathrm{N}$ & $\mathrm{XX}$ & $\mathrm{X}$ & . & 0 & $\mathrm{XX}$ & . \\
\hline & $28 \mathrm{R}-1,10-12$ & 293,90 & IIB & IS & $\mathrm{XX}$ & $\mathrm{X}$ & . & o & $\mathrm{XX}$ & . \\
\hline & $36 \mathrm{R}-1,50-53$ & 346,50 & IIB & $\mathrm{N}$ & $\mathrm{X}$ & $\mathrm{X}$ & $\mathrm{X}$ & $\mathrm{XX}$ & $\mathrm{X}$ & . \\
\hline & $36 \mathrm{R}-1,50-53$ & 346,50 & IIB & $\mathbf{S}$ & $\mathrm{X}$ & $\mathrm{X}$ & $\mathrm{XX}$ & $\mathrm{XX}$ & $\dot{x}$ & . \\
\hline & $40 R-2,36-39$ & 367,25 & IIB & $\mathrm{N}$ & $\mathrm{x}$ & $\mathrm{x}$ & $\mathrm{X}$ & $\mathrm{XX}$ & O & . \\
\hline & $42 R-3,38-40$ & 383,03 & IIB & IS & $\mathrm{X}$ & $\mathrm{X}$ & $\mathrm{XX}$ & $\mathrm{XX}$ & . & . \\
\hline & $44 \mathrm{R}-\mathrm{CC}, 0-3$ & 392,64 & IIB & $\mathbf{N}$ & $\mathrm{X}$ & $\mathrm{X}$ & $\mathrm{XX}$ & $\mathrm{XX}$ & $\therefore$ & . \\
\hline & $47 R-2,24-26$ & 405,74 & IIB & IS & $\mathrm{X}$ & $\mathrm{X}$ & O & $\mathrm{X}$ & $\mathrm{XX}$ & . \\
\hline & $47 \mathrm{R}-2,24-26$ & 405,74 & IIB & IS & $\mathrm{X}$ & $\mathrm{X}$ & $\mathrm{XX}$ & $\mathrm{XX}$ & 0 & . \\
\hline & 51R-1, 129-131 & 424,69 & IIB & IS & $\mathrm{X}$ & $\mathrm{X}$ & $\mathrm{O}$ & $\mathrm{X}$ & $\mathrm{XX}$ & . \\
\hline & 51R-1, 129-131 & 424,69 & IIB & IS & $\mathrm{X}$ & $\mathrm{X}$ & $\mathrm{XX}$ & XX & . & . \\
\hline & $67 \mathrm{R}-1,50-52$ & 548,90 & IIC & MS & $\mathrm{XX}$ & $\mathrm{X}$ & . & $\mathrm{XXX}$ & . &. \\
\hline
\end{tabular}

Note: Type of samples: $\mathrm{S}=$ soft mud; $\mathrm{IS}=$ indurated mud; $\mathrm{MS}=$ metamorphic mud; $\mathrm{N}=$ nodule

Clays $=$ chlorite and smectite; Relative abundance: $X=15-20 \%, O<10 \%$

and sulfide phases (Table 3). Proportions of detrital quartz, feldspar, and mica are unvarying throughout Unit I and Subunits IIA and IIB. In the lower part of the sequence at Hole 857C (Subunit IIB), the relative content of quartz and feldspar decreases, while the proportion of phyllosilicates, such as mica and clays, increases. The deposits forming Subunits IIA and IIB, in both Holes 857A and 857B, comprise abundant carbonate cement and concretions; from the top to the bottom of the holes, the concretions are made of $\mathrm{Mg}$-poor calcite, $\mathrm{Ca}$-rich dolomite, and $\mathrm{Mg}$-rich calcite. The maximum $\mathrm{Mg}$ concentration in the secondary carbonates occurs in the lower part of the Subunit IIA in both holes (Früh-Green et al., this volume).

A negative correlation between carbonate and clay mineral content was observed in the indurated sediments and concretions, as at Site 856. In both Holes 857A and 857C, a zonation in the partitioning of the secondary phases can be established, from $\mathrm{Mg}$-rich clay minerals (mainly chlorite) at the bottom, through $\mathrm{Mg}$-rich calcite, to Ca-rich dolomite at the top.

\section{Active Hydrothermal Discharge Site 858}

The hemipelagic turbiditic sediments at Site 858 were hydrothermally modified in situ. Hydrothermal input is best expressed at Holes $858 \mathrm{~B}$, within the vent field, by the presence of intercalated deposits of secondary silicates, carbonates, sulfates, and sulfides (Table 4). A few of these deposits, such as the pure hydrothermal precipitates, appear free of detrital components. These precipitates are represented by a gypsum and anhydrite layer (Sample 139-858B-2H-4, 74-75 $\mathrm{cm}$ ), authigenic quartz and chlorite- or corrensite-rich layers (Samples 139-858B-5H-3, 58-62 cm, and -6H-1,51-53 cm) (Pl. 1, Fig. 3), and a pyrite and smectite layer (Sample 139-858B-2H-3, 75-77 cm) (Pl. 1, Fig. 4).

In sediments from the other holes studied at Site 858, detrital quartz, feldspar, and mica are ubiquitous in variable proportions. Feldspar and mica are not apparent on the XRD diagrams of the bulk deposits because of the abundance of the dominant secondary miner- 
Table 4. Bulk mineralogic composition of the sediments and nodules from Site 858 estimated by X-ray diffraction analyses on powdered samples.

\begin{tabular}{|c|c|c|c|c|c|c|c|c|c|c|c|}
\hline Hole & $\begin{array}{l}\text { Core, section, } \\
\text { interval }(\mathrm{cm})\end{array}$ & $\begin{array}{l}\text { Depth } \\
\text { (mbsf) }\end{array}$ & Unit & Type & Quartz & Feldspar & Mica & Clays & Calcite & Sulfates & Pyrite \\
\hline \multicolumn{12}{|l|}{ 139-858A- } \\
\hline & $\begin{array}{l}2 \mathrm{H}-3,36-40 \\
2 \mathrm{H}-7,30-31\end{array}$ & $\begin{array}{r}5,76 \\
11,70\end{array}$ & I & $\mathrm{N}$ & $\begin{array}{l}x \\
x\end{array}$ & $\mathrm{x}$ & $x x$ & $\underset{x}{x x}$ & $\mathbf{x y}$ & · & 0 \\
\hline & $2 \mathrm{H}-7,30-31$ & $\begin{array}{l}11,70 \\
1178\end{array}$ & $\mathrm{~J}$ & $\mathrm{~N}$ & & . & . & & $\mathrm{XxX}$ & . & . \\
\hline & $2 \mathrm{H}-7,38-40$ & & & $\mathrm{~N}$ & $x \mathbf{x}$ & . & $x x$ & $x x$ & . & . & . \\
\hline & $2 \mathrm{H}-7,40-42$ & 11,80 & I & $\mathrm{N}$ & $\mathrm{x}$ & $\mathrm{x}$ & . & $\mathrm{x}$ & $\mathrm{xxx}$ & . & . \\
\hline & $3 \mathrm{H}-1,0-3$ & 11,90 & I & $\mathrm{N}$ & $x x$ & 0 & . & . & $x x x$ & . & . \\
\hline & $3 \mathrm{H}-1,58-60$ & 12,48 & 1 & $s$ & $\mathrm{x}$ & $\mathrm{x}$ & $\mathrm{x}$ & $x x$ & & $O(G)$ & $\mathrm{x}$ \\
\hline & $4 \mathrm{H}-7,15-17$ & 30,55 & IIA & $\mathrm{s}$ & $x x$ & 0 & $\mathrm{x}$ & $\mathrm{x}$ & $\mathrm{xx}$ & . & . \\
\hline & $5 \mathrm{H}-2,134-137$ & 33,74 & IIA & $\mathrm{N}$ & $\mathrm{x}$ & $\mathrm{x}$ & $\mathrm{x}$ & $x x$ & $\mathrm{x}$ & . & . \\
\hline & $5 \mathrm{H}-4,27-30$ & 35,67 & IIA & s & $\mathrm{x}$ & o & $x x$ & $\mathrm{xx}$ & $\mathrm{x}$ & $O(A \propto B)$ & . \\
\hline & $8 \mathrm{H}-2,57-59$ & 60,90 & IIC & $\mathrm{N}$ & $\mathrm{x}$ & 0 & $\mathrm{x}$ & $\mathrm{x}$ & $x x x$ & . & . \\
\hline & $11 \mathrm{X}-\mathrm{CC}, 12-14$ & 73,59 & IIC & $\mathrm{s}$ & $\mathrm{x}$ & $\mathrm{x}$ & $\mathrm{x}$ & $\mathrm{x}$ & $\mathrm{x}$ & (G) & $\mathrm{x}$ \\
\hline & $12 \mathrm{X}-\mathrm{CC}, 14-15$ & 81,94 & IIC & s & $\mathrm{x}$ & . & o & $\mathrm{x}$ & $x x x$ & . & . \\
\hline & $14 \mathrm{X}-\mathrm{CC}, 11-13$ & 101,11 & IIC & $\mathrm{N}$ & $\mathrm{x}$ & 0 & 0 & $x x x$ & $\mathrm{xx}$ & . & . \\
\hline & $18 X-2,132-134$ & 142,42 & пC & $\mathrm{s}$ & $\mathrm{x}$ & 0 & $\mathrm{x}$ & $x x$ & . & $\mathrm{XX}(\mathrm{G})$ & . \\
\hline & $27 \mathrm{X}-1,24-26$ & 226,97 & IIC & $\mathrm{s}$ & $\mathrm{x}$ & $\mathrm{x}$ & $\mathrm{x}$ & $\mathrm{xx}$ & . & $\mathrm{X}(\mathbf{G})$ & . \\
\hline & $31 X-2,3-5$ & 266,17 & ПIC & $\mathrm{s}$ & $\mathrm{x}$ & $\mathrm{x}$ & $\mathrm{x}$ & $x x x$ & . & 0 & o \\
\hline \multirow[t]{18}{*}{ 139-858B- } & & & & & & & & & & . & . \\
\hline & $1 \mathrm{H}-2,129-133$ & 2,79 & I & $\mathbf{s}$ & $\mathrm{x}$ & o & $\mathrm{x}$ & $\mathrm{x}$ & $\mathrm{xx}$ & . & . \\
\hline & $1 \mathrm{H}-4,108-112$ & 5,58 & 1 & s & $x x$ & $\mathrm{x}$ & $\mathrm{x}$ & $x x$ & . & . & . \\
\hline & $1 \mathrm{H}-5,34-36$ & 6,34 & I & s & $x x$ & o & $\mathrm{x}$ & $x x$ & $\mathrm{x}$ & . & . \\
\hline & $2 \mathrm{H}-1,97-99$ & 8,17 & 1 & $\mathrm{~s}$ & $\mathrm{x}$ & 0 & 0 & $\mathrm{xxxx}$ & . & . & - \\
\hline & $2 \mathrm{H}-2,45-47$ & 9.15 & I & s & $\mathrm{x}$ & $\mathrm{x}$ & $x x$ & XX & . & . & $\mathrm{x}$ \\
\hline & $2 \mathrm{H}-3,75-77$ & 10,95 & IV & $s$ & . & . & . & $x x x$ & . & $\mathrm{O}(\mathrm{G})$ & $x x x$ \\
\hline & $2 \mathrm{H}-3,126-129$ & 11,46 & IV & $\mathrm{S}-\mathrm{Br}$ & $\mathrm{x}$ & . & $x x$ & $\mathrm{xx}$ & . & $X(G+A)$ & . \\
\hline & $2 \mathrm{H}-3,126-129$ & 11,46 & IV & S-Ma & $\mathrm{x}$ & 0 & $x x$ & $x x$ & . & $X(G+A)$ & . \\
\hline & $2 \mathrm{H}-4,72-74$ & 12,42 & IV & $s$ & . & . & . & $\mathrm{xx}$ & . & $\operatorname{XXXXX}(G+A)$ & . \\
\hline & $2 \mathrm{H}-5,113-115$ & 14,33 & I & s & $x x$ & $\mathrm{x}$ & $\mathrm{x}$ & $x x x$ & $\mathrm{x}$ & $X(G)$ & 0 \\
\hline & $2 \mathrm{H}-6,89-91$ & 15,59 & 1 & $\mathrm{~s}$ & $x x$ & 0 & $x x$ & $\mathrm{XXX}$ & o & $O(\mathbf{G})$ & - \\
\hline & $5 \mathrm{H}-2,69-73$ & 26,09 & III & s & 0 & . & . & $\mathrm{XXXX}$ & . & . & . \\
\hline & $5 \mathrm{H}-3,58-62$ & 27,48 & ІІВ & $\mathrm{s}$ & $x x$ & . & . & $\mathrm{XXXX}$ & . & . & - \\
\hline & $5 \mathrm{H}-4,55-59$ & 28,95 & IIB & s & 0 & . & . & XXXXX & & . & . \\
\hline & $6 \mathrm{H}-1,51-53$ & 32,01 & IID & IS & $x X$ & . & . & XXXX & . & . & . \\
\hline & $6 \mathrm{H}-1,67-70$ & 32,17 & III & IS & $x$ & . & . & $\mathrm{x}$ & . & $\mathrm{XXX}(\mathrm{G}+\mathrm{A})$ & . \\
\hline & $8 X-1,27-31$ & 32,97 & IID & IS & $\mathrm{x}$ & . & . & $\mathrm{x}$ & . & $\mathrm{XXX}(\mathrm{G}+\mathrm{A})$ & . \\
\hline $159=858 \mathrm{C}$ & $2 \mathrm{H}-2,39-4 \mathrm{I}$ & 5,39 & 1 & $\mathrm{~N}$ & $\mathrm{x}$ & o & . & . & XXXX & . & . \\
\hline & $2 \mathrm{H}-5,56-58$ & 10,06 & I & IS & $\mathrm{x}$ & 0 & $\mathrm{x}$ & $x x$ & $\mathrm{x}$ & . & . \\
\hline & $2 \mathrm{H}-5,71-73$ & 10,30 & I & $\mathrm{s}$ & $\mathrm{x}$ & $\mathrm{x}$ & $\mathrm{x}$ & $x x$ & $\mathrm{x}$ & . & . \\
\hline & $2 \mathrm{H}-5,71-73$ & 10,30 & I & $\mathrm{N}$ & $\mathrm{x}$ & 0 & . & . & $\mathrm{XXXX}$ & . & . \\
\hline & $2 \mathrm{H}-6,81-83$ & 11,81 & IIB & s & $\mathrm{x}$ & $\mathrm{x}$ & $\mathrm{x}$ & $x$ & $\mathrm{xX}$ & . & . \\
\hline & $3 \mathrm{H}-2,100-115$ & 15,50 & IIB & $\mathrm{N}$ & . & . & . & . & $\mathrm{XXXXX}$ & . & . \\
\hline & $8 \mathrm{H}-\mathrm{CC}, 0-3$ & 47,17 & IIB & $\mathrm{N}$ & $x x$ & . & . & . & $\mathrm{XXX}$ & . & . \\
\hline \multicolumn{12}{|l|}{ 139-858D- } \\
\hline & $1 \mathrm{H}-1,72-75$ & 0,72 & 1 & IS & $\mathrm{x}$ & . & . & . & $\mathrm{XXXX}$ & . & . \\
\hline & $1 \mathrm{H}-5,75-77$ & 6,75 & I & $\mathrm{N}$ & $\mathrm{x}$ & o & & & $\mathrm{XXXX}$ & . & . \\
\hline & $2 \mathrm{H}-1,92-94$ & 10.22 & 1 & $s$ & $x x$ & $\mathrm{x}$ & $x$ & $\mathrm{x}$ & $\mathrm{X}(\mathrm{D})$ & . & . \\
\hline & $2 \mathrm{H}-2,44-46$ & 11,24 & I & IS & $x x$ & $\mathrm{x}$ & $\mathrm{x}$ & $\mathrm{x}$ & $X(D)$ & . & . \\
\hline & $2 \mathrm{H}-3,101-103$ & 13,31 & I & IS & $x x$ & $\mathrm{x}$ & $\mathrm{x}$ & $x$ & $X(D)$ & . & . \\
\hline & $2 \mathrm{H}-4,99-101$ & 14,79 & 1 & $\mathrm{~s}$ & $\mathrm{x}$ & 0 & 0 & $x x$ & $\mathrm{xx}$ & . & . \\
\hline & $2 \mathrm{H}-5,80-82$ & 16,10 & 1 & $\mathrm{~s}$ & $x x$ & o & 0 & $x x$ & $\mathrm{xx}$ & . & . \\
\hline & $4 \mathrm{H}-1,5-7$ & 19,85 & IIA & IS-N & $\mathrm{x}$ & o & . & . & $\mathrm{XXXX}$ & . & . \\
\hline & $4 \mathrm{H}-1,11-13$ & 19.91 & IIA & IS-N & $\mathrm{x}$ & o & & & $\mathrm{XXXX}$ & . & . \\
\hline & $4 \mathrm{H}-2,18-20$ & 20,22 & IIA & IS & $\mathrm{x}$ & $\mathrm{o}$ & $\mathrm{x}$ & $\mathrm{x}$ & $\mathrm{xx}$ & . & . \\
\hline & $4 \mathrm{H}-2,28-30$ & 20,32 & IIA & IS-N & $\mathrm{x}$ & o & o & o & $x x x$ & . & . \\
\hline & $4 \mathrm{H}-2,78-80$ & 20,78 & IIA & IS-N & $\mathrm{x}$ & o & 0 & 0 & $x x x$ & . & . \\
\hline & $4 \mathrm{H}-4,1-3$ & 23,05 & III & IS & $x x$ & o & $x x$ & $x x$ & . & . & . \\
\hline & $7 \mathrm{X}-\mathrm{CC}, 1-3$ & 3721 & III & IS-N & $\mathrm{x}$ & 0 & . & . & $\mathrm{XXXX}$ & . & . \\
\hline \multirow[t]{2}{*}{ 139-858F- } & & & & & & & & & & . & . \\
\hline & 2R-CC, 0-5 & 27,80 & IIA & $\mathrm{N}$ & $\mathrm{x}$ & 0 & . & . & $\mathrm{XXXX}$ & . & . \\
\hline
\end{tabular}

Sulfates: anhydrite (A), bassanite (B), and gypsum (G); Relative abundance: $X=15 \%, 0<10 \%$

als. Disseminated pyrite and marcasite occur mainly in Hole $858 \mathrm{~B}$ and less commonly in the distal drill holes. Among the sulfates, anhydrite and gypsum are the prevalent phases, but bassanite, which is a lowhydrated Ca-sulfate, was also detected (Hole 858A, at $35.67 \mathrm{mbsf}$ ). Calcite and dolomite are more common in sediments from distal Holes $858 \mathrm{~A}, 858 \mathrm{C}$, and $858 \mathrm{D}$. The close associations of authigenic minerals, such as phyllosilicate quartz and phyllosilicate sulfide, suggest that they represent hydrothermal parageneses related to the evolution of the original reacting fluid.

\section{Carbonate Concretions}

Among the secondary authigenic phases found in the modified sediments, the calcitic concretions record the most specific record of hydrothermal processes in the Middle Valley sequence. A summary of the mineralogy and isotope geochemistry of calcium carbonate concretions found in surficial deposits of the same area has been reported by Davis et al. (1987), Goodfellow and Blaise (1988), and Al-Aasm and Blaise $(1987,1991)$, among others. Concretions recovered at varying 
depths in the drill holes at Sites 856,857 , and 858 commonly consist of calcium carbonate impregnating and rarely replacing hemipelagic and turbiditic sediments. The concretions appear at various depths and their occurrence does not seem to be correlated with sediment compaction. In each hole, the concretions can be divided into various types according to their shape, texture, luminescence character, mineralogy, and major-element composition (Table 5). The stable isotope characterization of the concretions is given by Früh-Green et al. (this volume).

\section{Recrystallized Carbonate Nodules at Former Discharge Site 856}

Carbonate nodules recovered in Hole 856A, in lithologic Subunits IIA and IIC, are more common in coarser-grained turbidite beds than in clay-rich intervals. They are spherically shaped, rarely zoned, and in intergranular infillings typically show the same laminations and pyrite veinlets or framboidal aggregates as the surrounding sediments. A distinct boundary between the concretion and the sediment is generally present. The presence of the nodules is correlated with a decrease in the preservation of calcareous biogenic components.

Two main types of nodules are defined: (1) concretions related to low-carbonate precipitation in silty clays, which are chiefly found in the uppermost intervals, and (2) better-developed concretions hosted in the coarse silts. Intermediate types, forming moderately cemented concretions in finer intervals, are found in situ below $80.55 \mathrm{mbsf}$. The occurrence of different types of nodules appears to be related to the relative amount of carbonate and clay minerals (see "Bulk Sediment Mineralogy" section, this chapter, Table 2).

\section{Type 1}

In the fine silty, laminated, heavily burrowed sediment with calcite-poor cement, centimetric concretions at 37.08 and $40.31 \mathrm{mbsf}$ are generally elongated, slightly cemented, and unzoned; foraminifers are scarce. Cathodoluminescence observations show a scattering of dull red-luminescing points corresponding to the unzoned network of the cement.

\section{Type 2}

At approximately $46 \mathrm{mbsf}$ an abrupt change in the characteristics of the concretions occurs in the coarse silt intervals. Rounded and well-indurated nodules, about $3 \mathrm{~cm}$ in diameter or smaller, exhibit various and gradual stages of recrystallization, which commonly preserves the primary arrangement of clasts and pyrite grains and other sedimentary structures (Pl. 2). A trend of diagenetic enrichment in manganese of the recrystallized carbonates is roughly related to increasing depth (Table 5). In addition to these common features, each studied nodule appears to have specific textural microscopic characteristics that are more related to the original grain-size distribution of the host deposit and their secondary clay-mineral enrichment than to depth.

For example, the shallowest sampled nodule at 45.93 mbsf lacks structural zonation. The cement is homogenous, and incipient recrystallization of the microfossils is revealed by cathodoluminescence. The cement ranges from pure calcite to low- $\mathrm{Mg}$ calcite, with a relatively high Mn-content (Table 5). Lower Mn and higher Sr contents correspond to the unrecrystallized parts of the microfossil tests. A similar concretion, heavily cemented, was found within a deeper interval (on top of Subunit IIC at $95.84 \mathrm{mbsf}$ ). At $60.01 \mathrm{mbsf}$, a small concretion is formed of abundant cement made of large calcite crystals, up to $2 \mathrm{~mm}$, with variable extinction and a poikilotopic structure that is emphasized by catholuminescence (Pl. 2, Fig. 1). Within a similar nodule sampled at $73.38 \mathrm{mbsf}$, the cementing calcite crystals are more abundant and larger along the rim than in the center. A calcite vein with plumose fabric and undulating extinction (PI. 2, Fig. 2) was observed in a nodule sampled at $80.33 \mathrm{mbsf}$. It differs from the calcite cement by its higher red-orange luminescence; this difference cannot be explained by the slightly variable $\mathrm{Mn}$ contents of the $\mathrm{Fe}$-poor calcites (Table 5).

Samples recovered at $80.55,80.63$, and 109.47 mbsf define an intermediate type between type 1 and type 2 nodules that consist of poorly cemented silty clay. All of these intermediate types of concretions are clay-rich, as established by XRD analyses (Table 2).

\section{Magnesian Calcium Carbonates from Hydrothermal Reservoir Site 857}

Secondary carbonate concentrations were recovered from Holes 857A and $857 \mathrm{C}$, between 46 and 461 mbsf, in Subunits IIA and IIB. The concentrations consist not only of small subrounded nodules, but also of amoeboid- and crab-shaped calcite concentrations, of cement in the fine-grained turbidite beds, and of vein infillings, especially at greater depths. Between 76 and 101 mbsf, dolomite prevails instead of calcite (Table 3 ). In contrast to the true nodules from Site 856, these concentrations lack evidence of recrystallization, and the characteristics of the coexisting carbonates in cement and sediment are not very different.

The first type of nodule, such as those occurring in the uppermost deposits of Subunit IIA, consists of finely laminated fine silt or coarse silt pervasively cemented by fine-grained calcite. Throughout the sequence, calcite is also disseminated within the claystone. No fossils, zonation, or evidence of recrystallization were observed in these nodules. No clear luminescence was seen in the uppermost sample, whereas the deeper samples show very bright, uniformly distributed red-orange colors. In these luminescing cements, the $\mathrm{Mn}$ and $\mathrm{Fe}$ contents are high (Table 5), reaching $2.6 \%$ and $0.9 \%$, respectively (Sample 139-857C-9R, 50-51 cm). Calcite is also Mg-rich (FrühGreen et al., this volume). The same kind of nodule, with strong luminescing calcite cementing a coarse silt, occurs in a deeper interval at 276.24 mbsf.

An example of another type of carbonate concentration is found in the lower section of Subunit IIB, at 346.50 mbsf. It corresponds to a crab-like calcite concretion, possibly filling a fracture within a cemented, fine-grained clastic sediment.

\section{Manganese-rich Carbonate Concretions in Distal Zones of the Active Hydrothermal Field, Site 858}

The deposits at the "vent" Hole 858B are free of secondary carbonate mineralization because calcium is trapped in sulfates. Carbonate layers, nodules, and concretions occur in sediments from the more distal Holes $858 \mathrm{~A}, 858 \mathrm{C}$, and $858 \mathrm{D}$ where they are concentrated in Unit I (Hole 858A) and Subunits IIA and IIC (Holes 858A and 858D). The nodules show strong differences in shape, mineralogy, and geochemistry, including stable isotope composition (Früh-Green et al., this volume). The main groups are related to the lithology of the host sediment. Bulk mineralogical composition and microprobe data are listed in Tables 4 and 5.

\section{Concretions from Distal Hole $858 \mathrm{~A}$}

Two main types of concretions can be defined, mainly in relation to the lithology of the host deposits: type 1, found in Unit I sediments, and type 2, found in Unit II sequences. The first type of nodules sampled in Unit I (from 11.70 to $11.90 \mathrm{mbsf}$ ) is irregularly shaped, up to $4 \mathrm{~cm}$ long, and partly corresponds to filled burrows. The formation of this type of nodule seems to be guided by bioturbation fronts. The host sediment is a foraminifer-bearing silty clay pervasively impregnated by microcrystalline calcite. Thin articulated cracks and small veins are filled with larger calcite crystals. Macrocrystals also occur associated with pyrite microframboids in the foraminifer chambers. Both the micro- and macrocrystalline calcites exhibit a uniform bright-orange luminescence (Pl. 2, Figs. 3 and 4), with a faint zonation in the void fillings, whereas the unrecrystallized tests do not luminesce. 
Table 5. Mineralogic and chemical data obtained from cathodoluminescence and microprobe analyses on carbonate nodules from Sites 856,857 , and 858 .

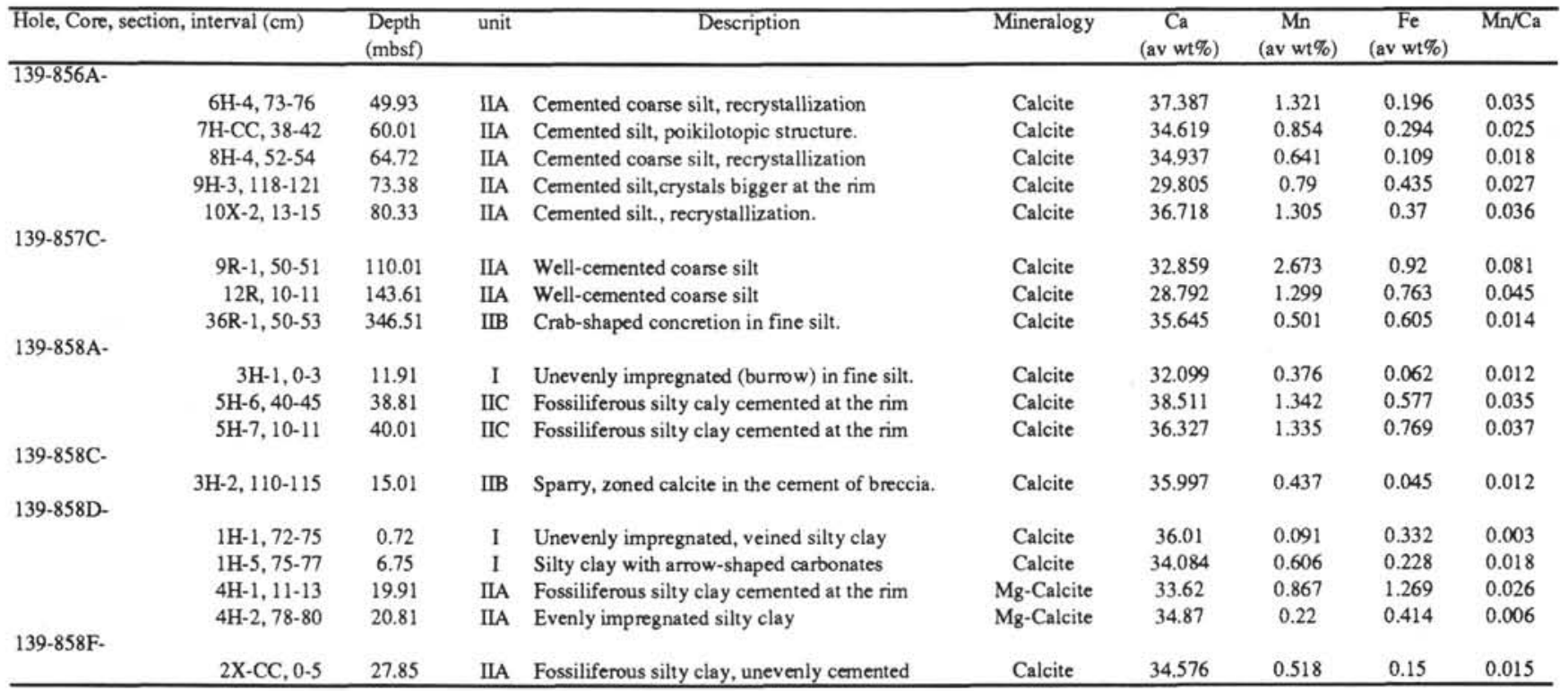

The type 2 nodules occur within Subunits IIA and IIC deposits and are characterized by a well-defined Mn-phase. They show different kinds of structure, and can be divided into three subtypes:

1. The first subtype is similar to the nodules from Unit I (in Core $139-858 \mathrm{~A}-5 \mathrm{H}$ at $32.42,38.80$, and $40.00 \mathrm{mbsf}$ ) in that it has a roughly concentric structure in which the size of the crystals increases toward the rim. The main difference with respect to the concretions from Unit I is that the former show a manganese enrichment. The rims are lined by Mn-oxide laminae. The carbonate fabrics range from a mosaic of crystals interspersed with Mn-oxides to coalescent dendroids. Common unaltered microfossil tests are nonluminescing; the secondary carbonates are orange luminescing and brighter at the border and in the sparry crystals infilling chambers (Pl. 3, Figs. 1 and 2). The luminescence is correlated with the Mn content; the tests are Mn-free, whereas $\mathrm{Mn}$ values up to 3.5 weight percent (wt\%) were measured in the sparry calcite.

2 . The second subtype of nodule is related to the poorly luminescing calcite that irregularly impregnates fine silt (samples at 30.55, 60.90 , and $64.61 \mathrm{mbsf}$ ). No crystalline pattern is visible by XRD and the carbonate spots were too irregular to be analyzed by microprobe.

3. The third subtype occurs at $33.74 \mathrm{mbsf}$ and corresponds to an encrustation of a coarser lithology, where abundant pyrite crystals and framboids occur within carbonate cement that luminesces dull red-orange.

\section{Concretions from Central Discharge Holes $858 \mathrm{C}$ and $858 \mathrm{D} / \mathrm{F}$}

A classification of the nodules is difficult to establish. Unit I at Hole $858 \mathrm{C}$ contains similar concretions but at a more incipient stage than those found in Unit I and the uppermost interval of Subunit IIA at Hole 858A. Ellipsoidal concretions (at 5.39, 10.06, and 47.17 mbsf) consist of fossiliferous silty clay cemented by microcrystalline calcite. Mn-oxide rims were observed in the deepest sample. Cathodoluminescence colors are patchily diffused in the sediment. The calcite is slightly magnesian (Früh-Green et al., this volume).

A concretion at 15.50 mbsf sampled below a more continuous layer of hydrothermal carbonates has a completely different shape consisting of a fragment of calcite cementing a breccia, small clasts, and dark silty clay. The fabric includes sparry, well-terminated crystals that are full of secondary fluid inclusions, commonly with a drusy structure. A bright orange luminescence shows faint visible zonation.
In the absence of significant Mn enrichment, this luminescence could reflect the low $\mathrm{Fe}$ content (Table 5).

In Hole $858 \mathrm{D}$, the sampled nodules can be divided between the type 1 found in surficial sediments at both Holes $858 \mathrm{~A}$ and $858 \mathrm{C}$ (Unit I) and the type 2 . The type 1 is represented by the subrounded concretion related to burrowing activity at $0.72 \mathrm{mbsf}$, which consists of orange-luminescing, Mn-poor microcrystalline calcite, and is cut by veinlets and cracks (Pl. 3, Fig. 3). The nodule recovered in the silty clay of Unit I at $6.75 \mathrm{mbsf}$ belongs to the type 2 concretions. The nodule is made of bright-red luminescing Mn-rich magnesian calcite in a pyrite-rich groundmass. Crystals are either dendroids or star-shaped arrows (Pl. 3, Fig. 4). Concretions found at 19.85, 19.91, 20.42, and 20.78 mbsf (Subunit IIA) show only subtle differences in shape (round to oval), degree of cementation, distribution and abundance of carbonates, and luminescence power (patchy reddish-orange to bright orange) which is a function of the Mn content. From these data, the general character of the carbonate concentrations as cements and nodules can be described:

Occurrence, concretion formation, and crystal growth are more favored within the coarse beds than in the clay-rich intervals, suggesting that porosity plays an important role in nodule formation as well as in the crystallinity of calcite.

Carbonate concentration is negatively correlated to the total clay content (from XRD data). Sediment induration may be the result of carbonate or clay neoformation.

The original texture of the enclosing sediment commonly is preserved, hence, cementation is a process superimposed on early burial.

Impregnation by carbonates typically follows early diagenetic processes such as the formation of framboidal pyrite and the hydrothermal precipitation of pyrite.

\section{Clay Mineral Assemblages}

\section{X-Ray-Diffraction Data}

The clay determination of the $<2-\mu \mathrm{m}$-fraction of each sample is summarized in Table 6 for Site 856 and Table 7 for Site 858 .

In both Holes 856A and 856B, mica, smectite, and chlorite are the major components of the clay fractions. These three components are present in sediments from Unit I that are unaffected by hydrothermal activity. In Hole 856A, smectite is dominant in the shallowest sample in Unit I. Deeper in the sedimentary column, samples from Subunit IIA contain "swelling" chlorite as a major component, i.e., chlorite 
Table 5 (continued).

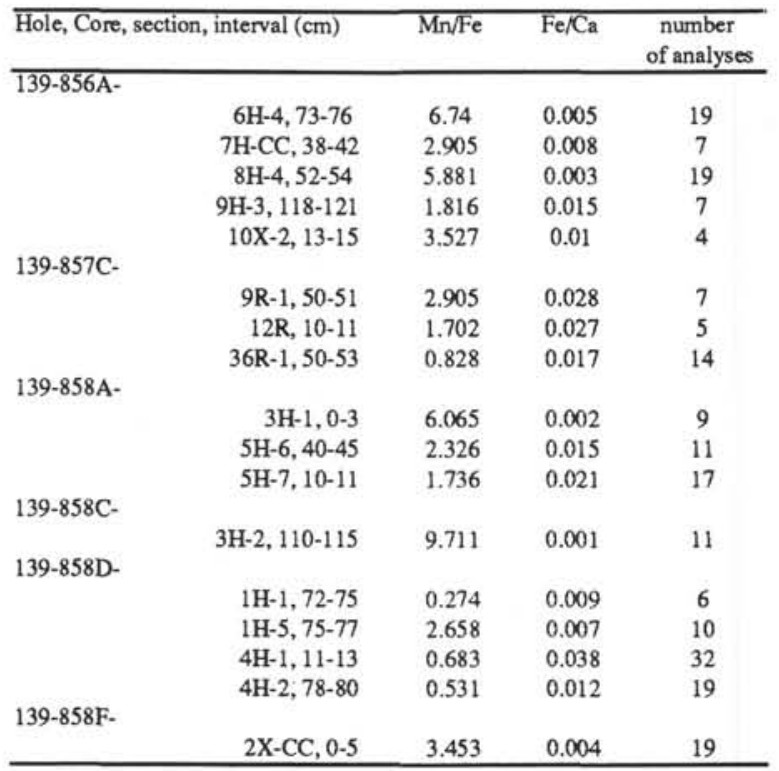

with some expandable layers. Below $100 \mathrm{mbsf}$, chlorite becomes abundant but smectite is still present. In Hole $856 \mathrm{~B}$, chlorite is abundant at all depths with mica and some smectite. Smectite occurs at various depths in this site. In general, at Site 856 , the clay assemblage does not seem to be related to lithology.

At Site 858 (Table 7), mica, smectite, and chlorite are also the dominant components of the clay fraction (Fig. 4A); however, corrensite, swelling chlorite, and talc are present in some samples. The assemblage here appears to be influenced by the nature of the lithologic units. In Unit I of each hole, mica, smectite, and chlorite prevail in the clay fraction. The fact that this unit represents unmodified sediments suggests that these clay minerals are detrital in origin. In Holes $858 \mathrm{~A}$, $858 \mathrm{C}$, and $858 \mathrm{D}$, mica is present at all depths in both unaltered and altered sediments. In Hole 858B, mica is absent in altered sediments (Unit IV and Subunits IIB and IIC).

A strong variation exists in the clay mineralogy of Hole $858 \mathrm{~B}$ sediments sampled at small intervals. The nature of the clays is clearly dependant on the lithologic unit (Fig. 4; Table 7). In Unit IV, a sample located at about $11 \mathrm{mbsf}$ is smectite rich (Fig. 4B). The diffraction pattern obtained for this sample shows that smectite is the only mineral in the clay fraction (Fig. 4B). The (060) reflection is at $1.533 \AA$, consistent with the trioctahedral structure of this clay mineral. One sample from Unit IV and samples from Subunit IIB contain a large proportion of corrensite. Corrensite appears in Sample 139-858B-2H$4,72-74 \mathrm{~cm}$, where it is mixed with chlorite; however, in Sample 139$858 \mathrm{~B}-5 \mathrm{H}-3,58-62 \mathrm{~cm}$, corrensite seems to be the only clay mineral in the $<2-\mu \mathrm{m}$ fraction. Random mixed-layer chlorite/smectite (or swelling chlorite) was also identified in samples from Units IV and IID.

Samples 139-858B-5H4, 55-59 cm, and 139-858B-6H-1, 51-53 $\mathrm{cm}$ (Fig. 4D) give a broad reflection between 10.9 and $9.8 \AA$ for the untreated clay fraction. This reflection becomes thinner and shifts to $9.2 \AA$ after glycolation and $9.5 \AA$ after heating. The reflection is difficult to assign; the fact that the (001) peak is broad could be explained by the size of the particles. Similar XRD patterns were described in Brindley and Brown (1980) for kerolite, which is an extremely fine-grained magnesium silicate that is chemically and structurally similar to talc. In order to test for the presence of kerolite in our samples, XRD patterns were obtained on coarser fractions ( 2 $\mu \mathrm{m}<\mathrm{X}<20 \mu \mathrm{m})$. The increase in the intensity of the peaks on the diffraction patterns shows that this mineral is more abundant in the larger-size fraction. It can be concluded, therefore, that this mineral is probably not kerolite. In fact, the broad reflection at about $9.8 \AA$ was
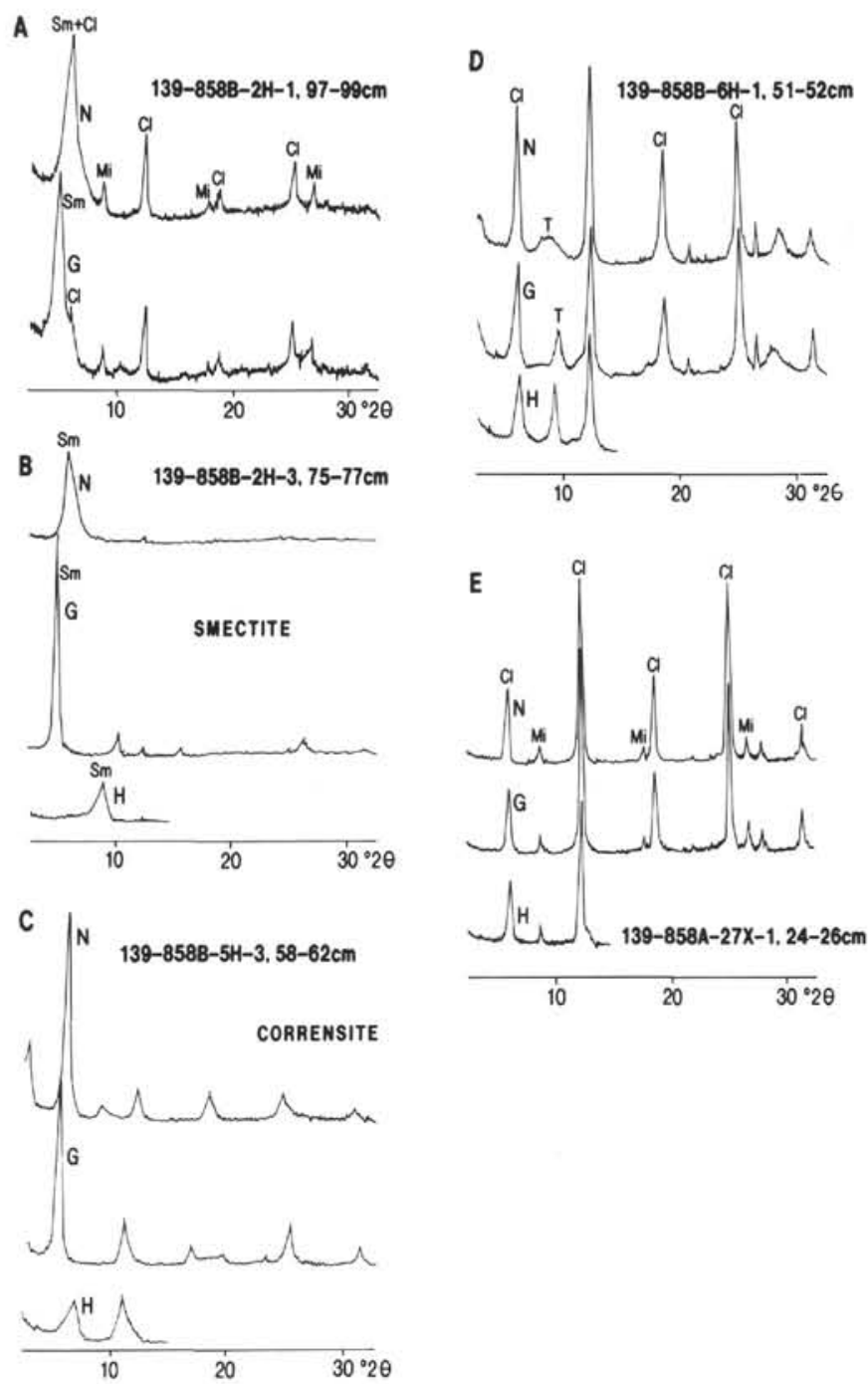

Figure 4. X-ray diffraction patterns of the $<-2 \mu \mathrm{m}$ clay fraction of samples from Site 858. A. Chlorite $(\mathrm{Cl})$, smectite $(\mathrm{Sm})$ and mica $(\mathrm{Mi})$ are present in this sample. B. Clay fraction is essentially composed of smectite. C. Corrensite is the major constituent of this clay fraction. D. Chlorite is dominant; however, "talc" (T) is also present in this sample (see discussion in text). E. Chlorite is a major constituent of the clay fraction, and mica is also present.

also detected in XRD patterns from disoriented bulk-sediment determinations; the other reflections recorded suggest that this mineral is talc. This hypothesis is confirmed by the high magnesium content of these samples (see Früh-Green et al., this volume). However, the presence of the broad reflection at $9.8 \AA$ is still not explained.

In Sample 139-858B-5H-2, 69-73 cm, corrensite is associated with a mineral that shows several reflections at $25,12.2$, and $8.25 \AA$. After glycolation these peaks move to $30,13.5$, and $8.8 \AA$. After heating at $490^{\circ} \mathrm{C}$ for $2 \mathrm{hr}$, the three peaks are observed at $12,9.4$, and $8 \AA$. According the data of Veniale and Van der Marel (1968), these reflections could correspond to allietite, a regular mixed-layer talc/saponite. Allietite is a rare mineral that has been described as an alteration product of serpentine; however, it seems to be a neoformed mineral with a hydrothermal origin. At $30 \mathrm{mbsf}$ in Hole $858 \mathrm{~B}$, chlorite becomes the prevalent mineral and is associated with corrensite or mixed-layer chlorite/smectite (Samples 139-858B-8X-2, 26-24 cm, and 858B-8X$4,22-24 \mathrm{~cm})$.

In Hole $858 \mathrm{~A}$, the section of Unit I located between 0 and about $30 \mathrm{mbsf}$ has the same assemblage as in Hole $858 \mathrm{~B}$, i.e., the clay 
Table 6. Mineralogic composition of the clay fraction of samples from Site 856 estimated from X-ray diffraction analyses on oriented particles.

\begin{tabular}{|c|c|c|c|c|c|c|c|c|c|}
\hline \multirow[b]{2}{*}{ Hole } & \multirow[b]{2}{*}{ Core, section, interval (cm) } & \multirow{2}{*}{$\begin{array}{l}\text { Depth } \\
\text { (mbsf) }\end{array}$} & \multirow[t]{2}{*}{ Unit } & \multicolumn{3}{|c|}{ Clay fraction composition } & \multirow[b]{2}{*}{$\begin{array}{l}\text { swelling } \\
\text { Chlorite }\end{array}$} & \multirow[b]{2}{*}{ Chlorite } & \multirow[b]{2}{*}{ Talc } \\
\hline & & & & Illite & Smectite & Corrensite & & & \\
\hline \multirow[t]{6}{*}{ T39-856A- } & THन1, 48-51 & 2.32 & $\mathrm{~T}$ & $\mathrm{X}$ & $\mathrm{XXX}$ & . & . & $\mathrm{XX}$ & $\cdot$ \\
\hline & $4 \mathrm{H}-1,36-40$ & 22.06 & IIA & $\mathrm{X}$ & $\mathrm{XX}$ & . & . & $\mathrm{XXX}$ & . \\
\hline & $6 \mathrm{H}-4,18-22$ & 45.38 & IIA & $\mathrm{XX}$ & . & . & $\mathrm{XXXX}$ & . & . \\
\hline & $6 \mathrm{H}-7,20-24$ & 49.9 & IIA & $\mathrm{X}$ & . & . & $\mathrm{XXXX}$ & . & . \\
\hline & $7 \mathrm{H}-7,49-52$ & 59.69 & IIA & $\mathrm{X}$ & . & $\mathrm{XXX}$ & . & $\mathrm{XX}$ & . \\
\hline & $13 \times 4,73-77$ & 110.53 & IIC & . & $\mathrm{X}$ & . & $\mathrm{XX}$ & $\mathrm{XX}$ & . \\
\hline \multirow[t]{4}{*}{ 139-856B- } & $2 \mathrm{H}-7,32-36$ & 11.12 & ILA & XXX & . & . & . & XXX & . \\
\hline & $5 \mathrm{H}-4,110-115$ & 35.9 & IIA & $\mathrm{XX}$ & $\mathrm{xx}$ & . & $*$ & $\mathrm{XXX}$ & . \\
\hline & $9 \mathrm{H}-2,74-77$ & 64.54 & IIC & $\mathrm{XX}$ & . & . & * & $\mathrm{XXXX}$ & . \\
\hline & $12 X-2,114-118$ & 84.24 & III & $\mathrm{x}$ & $x x$ & . & . & $\mathrm{XXX}$ & . \\
\hline
\end{tabular}

Table 7. Mineralogic composition of the clay fraction of samples from Site 858 estimated from X-ray diffraction analyses on oriented particles.

\begin{tabular}{|c|c|c|c|c|c|c|c|c|c|}
\hline \multirow[b]{2}{*}{ Hole } & \multirow[b]{2}{*}{ Core, section, interval (cm) } & \multirow{2}{*}{$\begin{array}{l}\text { Depth } \\
\text { (mbsf) }\end{array}$} & \multirow[t]{2}{*}{ Unit } & \multicolumn{3}{|c|}{ Clay fraction composition } & \multirow[b]{2}{*}{$\begin{array}{l}\text { swelling } \\
\text { Chlorite }\end{array}$} & \multirow[b]{2}{*}{ Chlorite } & \multirow[b]{2}{*}{ Talc } \\
\hline & & & & Illite & Smectites & Corrensite & & & \\
\hline \multirow[t]{10}{*}{$139-858 \mathrm{~A}-$} & $2 \mathrm{H}-3,36-40$ & 5.76 & $T$ & $\mathrm{XX}$ & $\mathrm{XX}$ &. & $\cdot$ & $\mathrm{XX}$ & $\cdot$ \\
\hline & $3 \mathrm{H}-1,58-60$ & 12.48 & I & $\mathrm{XX}$ & $\mathrm{XX}$ & . & . & $\mathrm{XX}$ & . \\
\hline & $4 \mathrm{H}-7,15-17$ & 30.55 & I & $\mathrm{XXXX}$ & $\mathrm{X}$ & . & . & $\mathrm{X}$ & . \\
\hline & $5 \mathrm{H}-4,27-30$ & 35.67 & IIA & $\mathrm{XXX}$ & . & . & $\mathrm{XXX}$ & . & . \\
\hline & $11 \mathrm{X}-\mathrm{CC}, 12-14$ & 73.59 & IIC & $\mathrm{XX}$ & . & . & $\mathrm{XXX}$ & $\mathrm{X}$ & . \\
\hline & $12 X-C C, 14-15$ & 81.94 & IIC & $\mathrm{XXX}$ & . & : & $\mathrm{XXX}$ & . & . \\
\hline & $14 \mathrm{X}-\mathrm{CC}, 11-13$ & 101.11 & IIC & $\mathrm{XX}$ & . & . & . & $\mathrm{XXXX}$ & . \\
\hline & $18 X-2,132-134$ & 142.42 & IIC & $\mathrm{XX}$ & . & . & . & $\mathrm{XXXX}$ & . \\
\hline & $27 X-1,24-26$ & 226.97 & IIC & $\mathrm{X}$ & . & . & . & $\mathrm{XXXXX}$ & . \\
\hline & $31 X-2,3-5$ & 266.17 & IIC & $\mathrm{X}$ & . & . & . & $\mathrm{XXXXX}$ & . \\
\hline \multirow[t]{12}{*}{$139-858 \mathrm{~B}-$} & $2 \mathrm{H}-1,97-99$ & 8.17 & I & $\mathrm{X}$ & $\mathrm{XXX}$ & . & . & $\mathrm{XX}$ & . \\
\hline & $2 \mathrm{H}-2,45-47$ & 9.15 & I & $\mathrm{XX}$ & $\mathrm{X}$ & . & . & $\mathrm{XXX}$ & . \\
\hline & $2 \mathrm{H}-3,75-77$ & 10.95 & IV & . & XXXXX & . & . & $\mathrm{X}$ & . \\
\hline & $2 \mathrm{H}-4,72-74$ & 12.42 & IV & . & . & XXXXX & $\mathrm{X}$ & . & . \\
\hline & $2 \mathrm{H}-5,113-115$ & 14.33 & 1 & $\mathrm{x}$ & $\mathrm{x}$ & . & $\mathrm{x}$ & $\mathrm{XXX}$ & . \\
\hline & $2 \mathrm{H}-6,89-91$ & 15.59 & I & $\mathrm{XX}$ & . & . & . & XXXX & . \\
\hline & $5 \mathrm{H}-2,69-73$ & 26.09 & IIB & . & . & XXXX & . & . & $\mathrm{XXA}$ \\
\hline & SH-3, $58-62$ & 27.48 & III & . & . & $\mathrm{XXXXXX}$ & . & . & . \\
\hline & $5 \mathrm{H}-4,55-59$ & 28.95 & IIB & . & . & $\mathrm{XXXXX}$ & . & . & $\mathrm{X}$ \\
\hline & $6 \mathrm{H}-1,51-53$ & 32.01 & III & . & . & . & $\mathrm{XX}$ & $\mathrm{XXXX}$ & $\mathrm{XX}$ \\
\hline & $8 X-2,26-28$ & 34.0 & III & , & $x$ & $\mathrm{XX}$ & . & $\mathrm{XXX}$ & . \\
\hline & $8 X-4,22-24$ & 36.3 & IID & . & $x$ & $\mathrm{XX}$ & . & $\mathrm{XXX}$ & . \\
\hline \multirow[t]{5}{*}{$139-858 \mathrm{C}$} & $7 \mathrm{H}-03,74-76$ & 45.24 & IIB & . & . & . & . & $\mathrm{XXXXXX}$ & . \\
\hline & $7 \mathrm{H}-04,3-6$ & 46.03 & IIB & . & . & . & . & $\mathrm{XXXXXX}$ & . \\
\hline & $8 \mathrm{H}-01,17-19$ & 46.67 & IIB & * & . & . & . & $\mathrm{XXXXXX}$ & . \\
\hline & $11 X-01,52-54$ & 55.02 & ПIC & . & . & . & . & $\mathrm{XXXXXX}$ & . \\
\hline & $13 \mathrm{X}-01,38-40$ & 74.08 & IIC & . & . & . & . & $\mathrm{XXXXXX}$ & . \\
\hline \multirow[t]{8}{*}{ 139-858D } & $1 \mathrm{H}-02,78-82$ & 2.28 & I & $x X$ & $\mathrm{XX}$ & . & . & $\mathrm{XX}$ & . \\
\hline & $1 \mathrm{H}-05,75-77$ & 6.75 & I & $x x$ & $\mathrm{XX}$ & . & . & $\mathrm{XX}$ & . \\
\hline & $1 \mathrm{H}-06,52-56$ & 8.02 & I & $\mathrm{XX}$ & $\mathrm{XX}$ & . & . & $\mathrm{XX}$ & . \\
\hline & $2 \mathrm{H}-\mathrm{CC}, 17-19$ & 18.99 & IIA & $\mathrm{XXX}$ & . & . & . & $\mathrm{XXX}$ & . \\
\hline & $4 \mathrm{H}-02,114-116$ & 21.18 & IIA & $\mathrm{XXX}$ & . & . & . & $\mathrm{XXX}$ & . \\
\hline & $4 \mathrm{H}-04,119-123$ & 24.23 & IIA & $\mathrm{XX}$ & . & . & . & $\mathrm{XXXX}$ & . \\
\hline & $4 \mathrm{H}-05,105-109$ & 25.59 & IIA & $\mathrm{XX}$ & . & . & . & $\mathrm{XXXX}$ & . \\
\hline & $5 \mathrm{H}-\mathrm{CC}, 11-13$ & 28.41 & IIA & $\mathrm{XXX}$ & . & . & . & $\mathrm{XXX}$ & . \\
\hline
\end{tabular}

fraction comprises smectite, mica, and chlorite. Hydrothermally altered samples from 30 to $80 \mathrm{mbsf}$ are rich in swelling chlorite, but mica is also present. The deepest samples of Hole 858A are chlorite rich (Fig. 4E). Typical (001) peaks at 14 and $7 \AA$ become more intense and thinner as depth increases, perhaps indicating an increasing size of the chlorite particles.

As shown in Table 7, the clay assemblages at Holes $858 \mathrm{C}$ and $858 \mathrm{D}$ are similar, with chlorite-rich samples present in the hydrothermally modified sediments.

To summarize, the XRD data on the clay fractions of the modified sediment and the hydrothermal deposits from Holes $858 \mathrm{~A}$ and $858 \mathrm{~B}$ display a distinct mineralogical sequence with depth. The shallowest samples are smectite-rich, and with increasing depth, corrensite becomes the dominant mineral, followed by swelling chlorite, and then chlorite. Chlorite/smectite random mixed-layer clays identified in Site 858 are chlorite rich, but random mixed layers with a high proportion of smectite layers were not observed in this site.

The $(060)$ reflections were studied in order to characterize the trior dioctahedral nature of the clay minerals. On the diffractograms presented in Figure 5, two major peaks are present: one corresponds to an $(060)$ reflection of $1.50 \AA$ and the other to a reflection of $1.53-1.54 \AA$. The intensity of $(060)$ of $1.50 \AA$ seems to be correlated with the presence of mica; indeed, in Sample 139-858B-5H-2, 69-73 $\mathrm{cm}$, which is mica-free, this peak is absent. The smectite-bearing sample (139-858B-2H-3, 75-77 cm) has an (060) reflection at 1.530 $\AA$. The corrensite-rich samples display an (060) peak at $1.537 \AA$ 

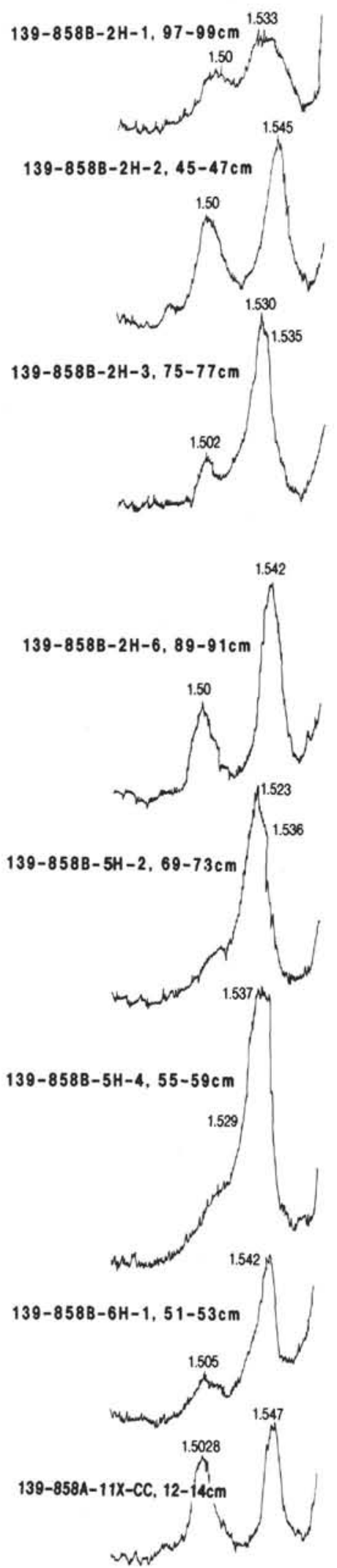

Figure 5. 060 reflections obtained on X-ray diffraction patterns of the clay fraction of samples from Site 858 .

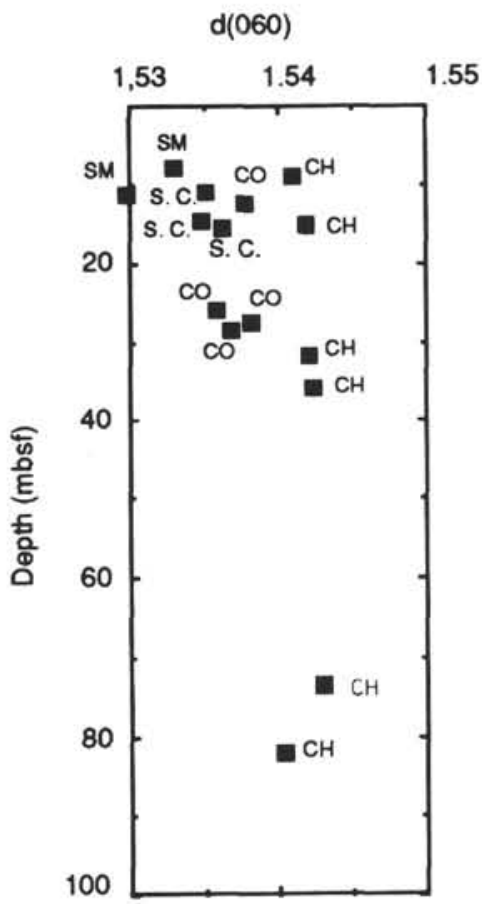

Figure 6. d(060) of the clays vs. depth for Holes $858 \mathrm{~A}$ and $858 \mathrm{~B}$. An increase in $\mathrm{d}(060)$ with depth is visible in this diagram, if we except the (060) of chlorite from the shallowest samples, that are probably detrital in origin. $\mathrm{SM}=$ smectite, $\mathrm{CO}=$ corrensite, $\mathrm{SC}=$ swelling chlorite, and $\mathrm{CH}=$ chlorite .

(Samples 139-858B-5H-4, 55-59 cm, and 858B-5H-2, 69-73 cm). Chlorite-rich samples (Samples 139-858B-6H-1,51-53 cm, and 139$858 \mathrm{~A}-27 \mathrm{X}-1,24-26 \mathrm{~cm})$ show an $(060)$ reflection at $1.542 \AA$. These results indicate that the mica is dioctahedral and that the chlorite and smectite are trioctahedral.

The plot of the $d(060)$ of the trioctahedral phases at Holes $858 \mathrm{~A}$ and $858 \mathrm{~B}$ vs. depth (Fig. 6) shows that the $\mathrm{d}(060)$, i.e., the $b$ value, increases with depth. The same trend has been observed by Inoue et al. (1984) in pyroclastic sediments from Japan, where interstratified chlorite/smectite are the intermediate products of a smectite-chlorite transition. This trend is generally explained by a progressive $\mathrm{Fe}$ for $\mathrm{Mg}$ substitution in the octahedral sites of the clay minerals.

\section{Morphologic characteristics of clay particles}

The observations of clay minerals by SEM and TEM allow smectite, corrensite, and chlorite to be distinguished by their morphologies. By SEM, Sample 139-858B-2H-3, 75-77 cm, shows particles that are curled thin sheets (Pl. 1, Fig. 4) characteristic of the authigenic smectites described in various oceanic sediments, especially those that have been altered by seawater or hydrothermal fluids (Karpoff, 1989). Corrensite, the major component of the clay fraction in Sample 139-858B$5 \mathrm{H} 3,58-62 \mathrm{~cm}$, has a different morphology typically with cornflakelike, slightly curled sheets (Pl. 4, Figs. 1 and 2). In contrast, the chlorite particles observed in Sample 139-858B-6H-1, 51-53 cm, appear as thicker rigid platelets about $2 \mu \mathrm{m}$ or more in diameter (PI. 4, Figs. 3 and 4). Chlorite platelets are intergrown with authigenic quartz crystals (Pl. 1, Fig. 3), indicating that chlorite is neoformed and cogenetic with the silica phase as a hydrothermal precipitate. In Sample 139-858B$8 \mathrm{X}-2,26-28 \mathrm{~cm}$, both corrensite and chlorite were observed in the same sample (Pl. 4, Figs. 2 and 3).

These various morphologies were also recognizable by TEM $(\mathrm{Pl}$. 5). In samples from Unit I at Sites 856 and 858, the heterogeneous clay assemblage is composed of mica particles, some quartz and feldspar fragments, and poorly crystallized smectite in thin particles with 
Table 8. Chemical analyses of the clay fraction of selected samples from Site 858 (wt \%).

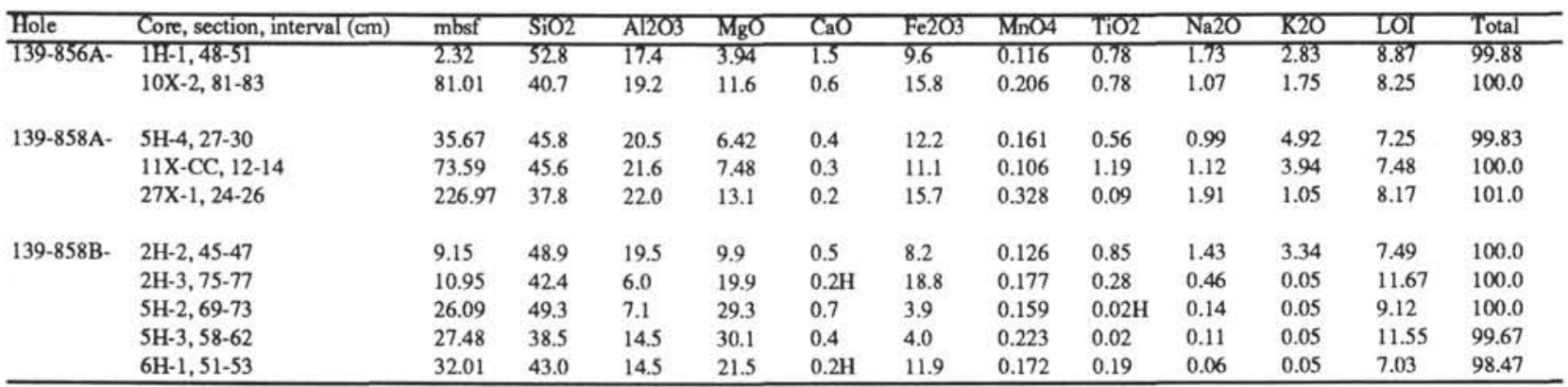

diffuse edges (Pl. 5, Fig. 1). The hydrothermal smectite from Sample $139-858 \mathrm{~B}-2 \mathrm{H}-3,75-77 \mathrm{~cm}$ occurs in large particles as crumpled veils with curled edges (PI. 5, Figs. 2 and 3). Neoformed chlorite (Sample $139-858 \mathrm{~B}-6 \mathrm{H}-1,51-53 \mathrm{~cm}$ ) displays well-shaped platelets with the mottled contrast typically observed for well-crystallized clay particles (Pl. 5, Fig. 4). Corrensite has an intermediate morphology between those of smectite flakes and chlorite platelets.

\section{Chemistry of the Clay Fractions}

Table 8 shows chemical analyses of the fine fraction for 10 samples from Sites 856 and 858 . These data display a relatively strong difference in composition among samples that reflects their mineralogical constitution. Samples 139-856A-1H-1, 48-51 cm, and 139$858 \mathrm{~B}-2 \mathrm{H}-2,45-47 \mathrm{~cm}$, both from Unit I and containing smectite, mica, and chlorite, have similar compositions with the exception that the $\mathrm{MgO}$ content is higher in the sample from Hole 858B. The sample from Site 856 can be considered a reference for background detrital clay in the sediments for comparison with the other samples composed of neoformed minerals. The composition of the clay fraction from Sample 139-858B-2H-3, 75-77 cm is fine trioctahedral smectite, which reflects the hydrothermal origin of the silicate: $\mathrm{MgO}$ is high (about $20 \%$ ) compared with the reference sample (4\% MgO). The high $\mathrm{Fe}$-content in this sample $\left(18 \% \mathrm{Fe}_{2} \mathrm{O}_{3}\right)$ can be explained by the presence of small pyrite grains in the clay fraction. The chemical composition of the clays in this sample (as well as in Samples 139-858B-5H-2, 69-73 cm, 858B-5H-3, 58-62 cm, and 858B-6H-1, $51-53 \mathrm{~cm}$ ) is probably related only to the hydrothermal fluids from which the clays precipitated, because no detrital minerals were detected. Samples 139-858B-5H-2, 69-73 cm, and 858B-5H-3, 58-62 $\mathrm{cm}$ are the most enriched in $\mathrm{MgO}(29-30 \%)$, whereas their $\mathrm{Fe}_{2} \mathrm{O}_{3}$ content is low (4\%). The chlorite in Sample 139-858B-6H-1, 51-53 $\mathrm{cm}$ is MgO-rich compared with the mixed-clay samples from Unit I, and $\mathrm{Fe}_{2} \mathrm{O}_{3}$-rich compared with the corrensite-bearing samples.

The fine fractions of Samples 139-856A-10X-2, 81-83 cm, 139$858 \mathrm{~A}-11-\mathrm{CC}, 12-14 \mathrm{~cm}$, and 858A-27X-1, 24-26 cm show high $\mathrm{Al}_{2} \mathrm{O}_{3}$ and $\mathrm{K}_{2} \mathrm{O}$ contents that reflect the presence of detrital mica mixed with swelling and/or authigenic chlorite.

The chemical influence of hydrothermal alteration on clay composition is evident on the ternary diagram $\mathrm{Fe}_{2} \mathrm{O}_{3}-\mathrm{MgO}-\mathrm{Al}_{2} \mathrm{O}_{3}$ (Fig. 7). The pure hydrothermal samples are enriched in $\mathrm{MgO}$ and $\mathrm{Fe}_{2} \mathrm{O}_{3}$, compared with the clay assemblages from Unit I. The compositions of samples containing a mixture of clays from different origins are highly influenced by the detrital component, and the hydrothermal signature is not well recorded.

\section{DISCUSSION}

\section{Origin of the Sediment Components}

In order to understand the mechanisms of formation of the hydrothermal products and to characterize the interaction between sedi- ments and hydrothermal fluids, it is necessary to discuss first the origin of these sediments to discriminate the detrital input from the neoformed minerals.

With only minor exceptions, the structure of the sedimentary successions in Middle Valley indicates a relatively simple depositional history, in which the sedimentation rate has generally kept pace with subsidence (Davis and Villinger, 1992). Unaltered sediments of the turbidite units contain detrital minerals with variable biogenic components (Goodfellow and Blaise, 1988). The finest sediments, silty clay grading to hemipelagic types, consist of a biogenic and terrigenous mixture, with local concentrations of diagenetic carbonates. According to Blaise et al. (1985) and Al-Aasm and Blaise (1991), the mineralogical composition of the finest clay fraction shows evidence of a variation between Pleistocene and Holocene deposits, owing to differences in continental weathering under variable climatic conditions. In sediments from the top of Unit I, mica, smectite, and chlorite coexisting with detrital quartz and feldspars are probably the result of continental weathering. Quartz, feldspar, and mica are present in surficial Unit I but are also abundant in the altered sediment of Site 856 and Holes $858 \mathrm{~A}, 858 \mathrm{C}$, and $858 \mathrm{D}$. The variation of the chemistry of feldspar was not investigated in this study. However, pore-water geochemical data display a decrease of $\mathrm{Ca}$ with depth that can be explained by albitization of feldspars. Furthermore, alteration and transformation of feldspar into albite, prehnite, and zeolite was observed in igneous rocks from Site 858 (Davis, Mottl, Fisher, et al., 1992).

All quartz identified in the deepest deposits of Hole 858B occurs as typical hexagonal euhedral shapes with very clean surfaces that reflect their neoformation. Their hydrothermal origin is established by a temperature of formation of approximately $270^{\circ} \mathrm{C}$ at $32 \mathrm{mbsf}$, calculated from stable isotope data (Früh-Green et al., this volume). Similar euhedral quartz is common in seafloor hydrothermal deposits, such as those found in surficial oceanic crust and associated pelagic sediments (Stoffers et al., 1985; Karpoff, 1989, 1992). Most of the deposited layers of Hole $858 \mathrm{~B}$ are free of detrital phases. Newly formed anhydrite and pyrite were also observed in shallower pure hydrothermal deposits.

\section{Clay Minerals}

The origin of the clays is more difficult to determine because it cannot be deduced solely by the morphology of the minerals in SEM or TEM images. However, TEM observations combined with chemical data allow the neoformed and detrital clays to be distinguished. These data were confirmed by stable isotope data from the same samples (Früh-Green et al., this volume). TEM observations of the clay fraction from the unmodified sediments (Unit I) display heterogeneous particles with different shapes. These clays are characterized chemically by high $\mathrm{Al}$ content in comparison with $\mathrm{Fe}$ and $\mathrm{Mg}$ contents. In contrast, the clay fraction of the deepest sediment from Hole 858B is homogeneous in TEM images, and authigenic smectite, observed only at this site, exhibits a thin curled sheet morphology that is different from that 
of the smectite in detrital deposits. The high $\mathrm{Mg}$ and low $\mathrm{Al}$ contents of this pure clay fraction, which is free of detrital contribution, confirm its hydrothermal origin. The hydrothermal samples from deeper in the hole show the same characteristics and are composed of one or two types of Mg-rich clay minerals. These results are confirmed by stable isotope data (Früh-Green et al., this volume). The samples from the other holes at Site 858 are composed of a mixture of hydrothermal clays with detrital minerals. Indeed, in the altered sediments from various depths, detrital mica, quartz, and feldspar coexist with hydrothermal chlorite or swelling chlorite. The chemical data on the clay fraction reflects an intermediate composition between hydrothermal and detrital clays.

From these data, the processes of formation of the hydrothermal clays can be proposed. Pure hydrothermal deposits occur only in Hole $858 \mathrm{~B}$, which is the closest hole to an active hydrothermal vent. Because of proximity to the vent, the fluid/rock ratio is probably higher in this area than in the other holes at Site 858. The occurrence of these pure hydrothermal deposits, and the fact that some of them are interbedded with unaltered sediments, suggest that a lateral circulation of fluids occurred in this area and that precipitation followed a metasomatic mechanism. In the other holes, the fluids partly transform the sediments: detrital clays become unstable and new hydrothermal phases precipitate from high-temperature fluids. However, quartz, feldspar, and mica are still stable in this environment, although the albitization of feldspars and slight corrosion and recrystallization of quartz occur in the deepest sediments (Davis, Mottl, Fisher, et al., 1992).

\section{Carbonate Concretions}

The carbonate concretions specifically record the hydrothermal processes occurring in sediments from Middle Valley. Secondary precipitation and recrystallization occurring within oxidizing zones use either calcium released from dissolution of biogenic phases or calcium supplied from deep hydrothermal fluids. The effect of convective circulation of fluids varying in temperature and composition superimposed on the heterogeneities such as grain-size distribution, porosity, and mineralogy of the host sediments, as well as local disturbance due to drilling (Davis, Mottl, Fisher, et al., 1992) could explain the apparent variations in the distribution and compositions of the nodules and concretions.

At each site, the concretions have peculiar characteristics that can be related to local hydrothermal conditions, such as the temperature gradient and/or the composition of the fluids and their evolution through space and time.

\section{Site 856}

Formation of the nodules results from strong modification of porefluid conditions. Concretions prevail within and below a sedimentary interval (near $40 \mathrm{mbsf}$ ) that was altered by the lateral flow of hydrothermal fluids, as recorded by the chemical compositions of the sediments and pore waters, and by isotopic trends (Davis, Mottl, Fisher, et al., 1992; Früh-Green et al., this volume). The late incursion of such thermal fluids into the sedimentary pile reactivates dissolutionprecipitation processes, causing changes in the equilibrium conditions of the various phases (primary phases and earlier hydrothermal products), which induce a remobilization of the elements and formation of a later generation of hydrothermal phases. For example, Mn enrichment of the deeper concretions probably results from downward migration of elements into more oxidized zones where conditions are favorable for precipitation.

\section{Site 857}

The concretions are uniform, unzoned, unrecrystallized, and in places $\mathrm{Mg}$ rich. Their formation is related mainly to thermal features

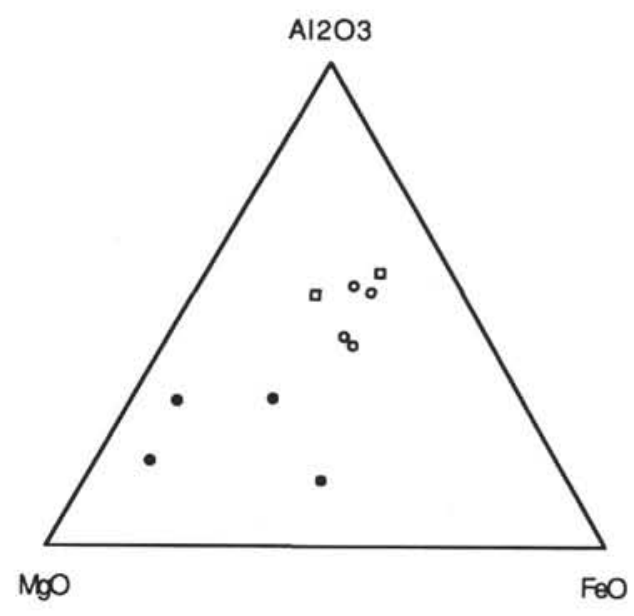

Figure 7. Ternary $\mathrm{MgO}-\mathrm{Al}_{2} \mathrm{O}_{3}-\mathrm{Fe}_{2} \mathrm{O}_{3}$ diagram (wt\%) showing the distribution of clay analyses. Pure hydrothermal samples are shown by filled circles (sample from Unit II of Hole 858B), detrital clays (samples from Unit I of Hole $858 \mathrm{~A}$ and $858 \mathrm{~B}$ ) by open squares, and mixtures of detrital mica and hydrothermal clays by open circles (samples from Hole 858A).

of high heat flow in this zone (Davis, Mottl, Fisher, et al., 1992). Increasing induration of the deposits downhole progressively leads to a closed system. At greater depths, the clay-rich intervals become unsuitable for later carbonate precipitation because of their low porosity and permeability, and act to restrain the circulation of highly concentrated fluids (Davis, Mottl, Fisher, et al., 1992), but not the vertical thermal diffusion. Local reduction-oxidation gradients that could occur below the Mg-rich carbonate intervals (such as between 75 and 102 mbsf in both Holes $857 \mathrm{~A}$ and $857 \mathrm{C}$, and about $143 \mathrm{mbsf}$ in Hole $857 \mathrm{C}$ ), seem to promote the formation of Mn-rich carbonates.

\section{Site 858}

Homogenous calcite-rich concretions occur only in the distal holes and in surficial deposits of the intermediate holes to the hydrothermal vent, which are farther from the influence of the more reduced central discharge zone. The concretions are distinguished by the presence of well-developed Mn-oxides or oxyhydroxides. At the sample scale, the paragenetic sequence is obvious: primary biogenic calcite, early diagenetic sulfides, sulfides, secondary calcite (generally Mn-rich), and Mn-oxides. Progressive induration is related to the transition from smectite to chlorite downhole. As at the other sites, the more clay-rich intervals are less calcareous (considering the secondary phases). Waterrock interactions are controlled by both the temperature and chemistry of the fluids.

\section{Mineralogical Sequence}

Based on investigations of altered sediments and hydrothermal deposits recovered in Site 858, a mineralogical sequence with depth can be established. This sequence may be related to the chemistry of fluids from which the silicate and carbonate phases precipitated, and to the thermal gradient. In Hole $858 \mathrm{~B}$, from the bottom to the top, $\mathrm{Si}$-rich deposits (quartz and clays) are covered by layers of pure $\mathrm{Mg}$-rich clay minerals that are overlain by calcium sulfate. On the top, iron sulfide and smectite-rich deposits are present. In distal Holes $858 \mathrm{~A}, 858 \mathrm{C}$, and $858 \mathrm{D}, \mathrm{Mg}-\mathrm{Fe}$ chlorite prevails at the bottom of the core, and calcium carbonate concretions with local Mn-oxides are developed in the uppermost deposits. The geochemical study of hydrothermal precipitates (Früh-Green et al., this volume) and of pore fluids demonstrate that a large-scale convective circulation can be defined at Site 858, with local recharge of cool water at Holes 858A 
and $858 \mathrm{C}$, and discharge proximal to Holes $858 \mathrm{~B}$ and $858 \mathrm{D} / \mathrm{F}$. In most of the holes, the stable isotope data on the shallow concretions suggest that the carbonate phases precipitated in an oxidizing environment, whereas most of the nodules that formed below 5 mbsf are generated by more reducting fluids. The oxidation conditions at shallow depths may be attributed to local inflow of cooler seawater (see Fig. 7 in Früh-Green et al., this volume).

In Hole $858 \mathrm{~B}$, mineralogic zonation is well expressed in the clay mineral assemblages. The lowermost deposits are chlorite rich, succeeded by corrensite as the dominant mineral, and followed by saponite-rich deposits in the uppermost layers. In Hole 858A, which is farthest from the vent, swelling chlorite is abundant in the uppermost altered samples, and chlorite becomes dominant with increasing depth. In each hole at Site 858 , smectite disappears as depth increases. This mineralogic sequence reflects a thermal gradient with depth. Stable isotope data obtained from neoformed clays confirm the occurrence of a geothermal gradient of $10^{\circ} \mathrm{C} / \mathrm{m}$ in Hole $858 \mathrm{~B}$ (Früh-Green et al., this volume). Furthermore, temperatures calculated from oxygen isotope fractionation between quartz and water and between chlorite and water are close to that measured in situ in the sediments, i.e., $200^{\circ} \mathrm{C}$ at about 20 mbsf (Davis, Mottl, Fisher, et al., 1992; Früh-Green et al., this volume). At Hole $858 \mathrm{~B}$, the clay sequence is also correlated with the geochemical gradient of pore water, i.e., $\mathrm{Mg}$ content decreases at 14 mbsf in the pore water of the deposits that are composed of $\mathrm{Mg}$-rich clay minerals (Davis, Mottl, Fisher, et al., 1992).

The observed clay mineral sequence at Middle Valley is similar to the smectite-corrensite-chlorite sequences described in other oceanic environments. Smectite-rich chlorite/smectite random mixed layers were not identified, but the occurrence of swelling chlorite indicates that chlorite-rich random chlorite/smectite or corrensite/chlorite mixed layers are present. This kind of sequence has been described in carbonate and evaporitic sequences, in soils, in clastic and volcaniclastic sequences, and in hydrothermal deposits (references in Shau and Peacor, 1992). Corrensite formation is generally ascribed to the conversion of smectite to chlorite by hydrothermal, diagenetic, or metamorphic processes. Most of the studies on trioctahedral phyllosilicate assemblages report an apparently prograding sequence similar to that observed in the Juan de Fuca sediments. Inoue et al. $(1984,1991)$ studied the conversion of smectite to chlorite in pyroclastic and volcaniclastic sediments and showed that the expandability of the clay minerals decreases from $100 \%-80 \%$ to $50 \%-40 \%$, and to $15 \%-10 \%$ in the more altered samples. These authors suggested two stages of reaction: (1) a transition of smectite to corrensite, and (2) a transition from corrensite to chlorite.

Similar parageneses of trioctahedral phyllosilicates in the transition zone and in the sheeted dike zone of Hole 504B are described by Shau and Peacor (1992). Corrensite, chlorite, and saponite/chlorite random mixed layers are present in this peculiar hydrothermal oceanic environment. Shau and Peacor (1992) found mixtures of chlorite/corrensite and talc/chlorite to be metastable products that occur only when the formation of relatively stable discrete phases is suppressed during rapid crystallization or during formation at low temperatures, or in the absence of a significant amount of fluid.

In the Juan de Fuca hydrothermal deposits, the prograding sequence of trioctahedral phyllosilicates could be due to a progressive transformation of a primary smectite directly precipitated from hydrothermal fluids, or to a direct precipitation of stable phases and metastable mixtures of smectite, corrensite, and chlorite. The first hypothesis requires the transformation of smectite to chlorite by outward migration of hydrothermal fluids that mix with pore waters and downward diffusing seawater. The zone of mixing would move outward, creating a prograde sequence with depth in the sediments (Davis, Mottl, Fisher, et al., 1992). However, the occurrence of a pure hydrothermal layer with well-differentiated mineralogic composition and geochemistry, and the fact that some hydrothermal deposits are intercalated with unmodified sediments, suggest that these hydrothermal products formed from laterally circulating fluids and by direct precipitation by a metasomatic mechanism. Achange in the fluid compositions is recorded by the mineral zonations and by a change in the composition of the pore fluids.

From these data, the physical and chemical role of the sediments in favoring precipitation of the hydrothermal phases can be discussed. In the active discharge zones, the sediments do not seem to participate chemically in the direct precipitation of the silicate minerals. However, in distal holes at Sites 858 and 856, where the sediments are less modified, biogenic components of the sediments contribute to the formation of carbonate concretions and newly formed clays may be the product of hydrothermal replacement of pre-existing phyllosilicates. In most cases, the sediments play an important role in the mode of precipitation of hydrothermal phases by facilitating the lateral circulation of fluids that form clay-cemented layers and the precipitation of carbonate in porous environments.

\section{CONCLUSIONS}

This mineralogic and petrographic study of the sediments from Middle Valley on the Juan de Fuca Ridge provides constraints on the origin of the silicate and carbonate phases and their mechanisms of formation. The unmodified sediments, which represent background sedimentation, are a mixture of detrital minerals and biogenic components. The neoformed minerals can be clearly distinguished by their morphology and composition. Hydrothermal clays are $\mathrm{Mg}$-rich compared with the detrital ones. Carbonate $(\mathrm{Ca}-\mathrm{Mg})$ concretions generally follow the clays and are the result of secondary precipitation using $\mathrm{Ca}$ released by the dissolution of biogenic phases and/or $\mathrm{Ca}$ supplied by hydrothermal fluids. These concretions preferentially form in more porous layers.

The distribution of the hydrothermal products in the sediments displays a mineralogic zonation that is especially well expressed at Site 858. Deposits from Hole 858B, closer to the active hydrothermal discharge, contain a mineralogic sequence, from the top to the bottom of the core, of pyrite-smectite, followed by Ca-sulfate, pure $\mathrm{Mg}$-clay layers, and quartz-chlorite assemblages. A mineralogic sequence that can be viewed as an expression of chemical "chromatography" of the intrusive hydrothermal solutions, is also revealed in the neoformed clay assemblage, which follows a temperature gradient upward of chlorite, swelling chlorite, corrensite, and smectite. The other holes have authigenic chlorite at the bottom and carbonate-rich layers at various depths.

The presence of pure hydrothermal deposits in Hole $858 \mathrm{~B}$ can be explained by a high flux of hot hydrothermal fluids invading the clastic sediments, followed by a metasomatic reaction. Precipitation occurred in a reducting environment in this axial zone, as is evident by the chemistry of the neoformed phases. The mineralogic zonation with depth most likely reflects the prevailing temperature gradient and the chemical evolution of the fluids from which the phases precipitated. In the more distal holes of Site 858 and in Site 856, the shallowest carbonates formed in an oxidizing environment probably due to more effective recharge by seawater. In these holes, hydrothermal fluid circulation appears weaker and more lateral, partially preserving the clastic deposits.

\section{ACKNOWLEDGMENTS}

The authors acknowledge J.R. Walker, D.R. Peacor, and J.F. Slack for their critical reviews of the manuscript. This study was supported by INSU grant, program “Geoscience Marine," contribution N913925 to M.D.B. and A.M.K.; ETH grant $\mathrm{N}^{\circ} 0-20-014-90$ to G.F.G.; and Swiss NSF grant $\mathrm{N}^{\circ} 20-29-52.90$ to J.A.M. 


\section{REFERENCES}

Al-Aasm, I.S., and Blaise, B., 1987. Hydrothermal fluids effect on the sediment column in Middle Valley, Juan de Fuca Ridge (N.E. Pacific). AAPG Program Abstr., 68:113.

, 1991. Interaction between hemipelagic sediment and a hydrothermal system: Middle Valley, northern Juan de Fuca Ridge, subarctic northeast Pacific. Mar. Geol., 98:25-40.

Besnus, Y., and Rouault, R., 1973. Une méthode d'analyse de roches au spectromètre d'arc à lecture directe par un dispositif d'électrode rotative. Analusis, 2:111-116.

Blaise, B., Bornhold, B.D., Maillot, H., and Chamley, H., 1985. Observations sur les environnements recents au nord de la dorsale Juan de Fuca (Pacifique Nord-Est). Rev. Geol. Dyn. Geogr. Phys., 26:201-203.

Brindley, G.W., and Brown, G. (Eds.), 1980. Crystal Structures of Clay Minerals and Their X-ray Identification. Mineral. Soc. London Monogr., 5.

Davis, E.E., Goodfellow, W.D., Bornhold, B.D., Adshead, J., Blaise, B., Villinger, H., and Le Cheminant, G.M., 1987. Massive sulfides in a sedimented rift valley, northern Juan de Fuca Ridge. Earth Planet. Sci. Lett., 82:49-61.

Davis, E.E., and Lister, C.R.B., 1977. Tectonic structures on the Juan de Fuca Ridge. Geol. Soc. Am. Bull., 88:346-363.

Davis, E.E., Mottl, M.J., Fisher, A.T., et al., 1992. Proc. ODP, Init. Repts., 139: College Station, TX (Ocean Drilling Program).

Davis, E.E., and Villinger, H., 1992. Tectonic and thermal structure of the Middle Valley sedimented rift, northern Juan de Fuca Ridge. In Davis, E.E., Mottl, M.J., Fisher, A.T., et al., Proc. ODP, Init. Repts., 139: College Station, TX (Ocean Drilling Program), 9-41.

Eberhart, J.P., 1989. Analyse Structurale et Chimique des Matériaux: Paris (Dunod Ed.).

Goodfellow, W.D., and Blaise, B., 1988. Sulfide formation and hydrothermal alteration of hemipelagic sediment in Middle Valley, northern Juan de Fuca Ridge. Can. Mineral., 26:675-696.
Inoue, A., and Utada, M., 1991. Smectite-to-chlorite transformation in thermally metamorphosed volcaniclastic rocks in the Kamikita area, northern Honshu, Japan. Am. Mineral., 76:628-640.

Inoue, A., Utada, M., Nagata, H., and Watanabe, T., 1984. Conversion of trioctahedral smectite to interstratified chlorite/smectite in Pliocene acidic pyroclastic sediments of the Ohyu district, Akita Prefecture, Japan. Clay Sci., 6:103-116.

Karpoff, A.M., 1989. Les faciès pélagiques condensés Cénozoiques des océans Pacifique et Atlantique: témoins des grandes crises géodynamiques [Ph.D. dissert.]. Univ. Louis Pasteur, Strasbourg.

, 1992. Cenozoic and Mesozoic sediments from the Pigafetta Basin, Leg 129, Sites 800 and 801: mineralogical and geochemical trends of the deposits overlying the oldest oceanic crust. In Larson, R.L., Lancelot, Y., et al., Proc. ODP, Sci. Results, 129: College Station, TX (Ocean Drilling Program), 3-30.

Shau, Y.H., and Peacor, D.R., 1992. Phyllosilicates in hydrothermally altered basalts from DSDP Hole 504B, Leg 83. A TEM and AEM study. Contrib. Mineral. Petrol., 112:119-133.

Stoffers, P., Lallier-Verges, E., Pluger, W., Schmitz, W., Bonnot-Courtois, C., and Hoffert, M., 1985. A "fossil" hydrothermal deposit in the South Pacific. Mar. Geol., 62:133-151.

Veniale, F., and Van der Marel, H.W., 1968. Aregular talc-saponite mixed-layer mineral from Ferriere, Nure Valley (Piacenza Province, Italy). Contrib. Mineral. Petrol., 17:237-254.

- Abbreviations for names of organizations and publications in ODP reference lists follow the style given in Chemical Abstracts Service Source Index (published by American Chemical Society).

Date of initial receipt: 2 December 1992

Date of acceptance: 9 January 1993

Ms 139SR-216 

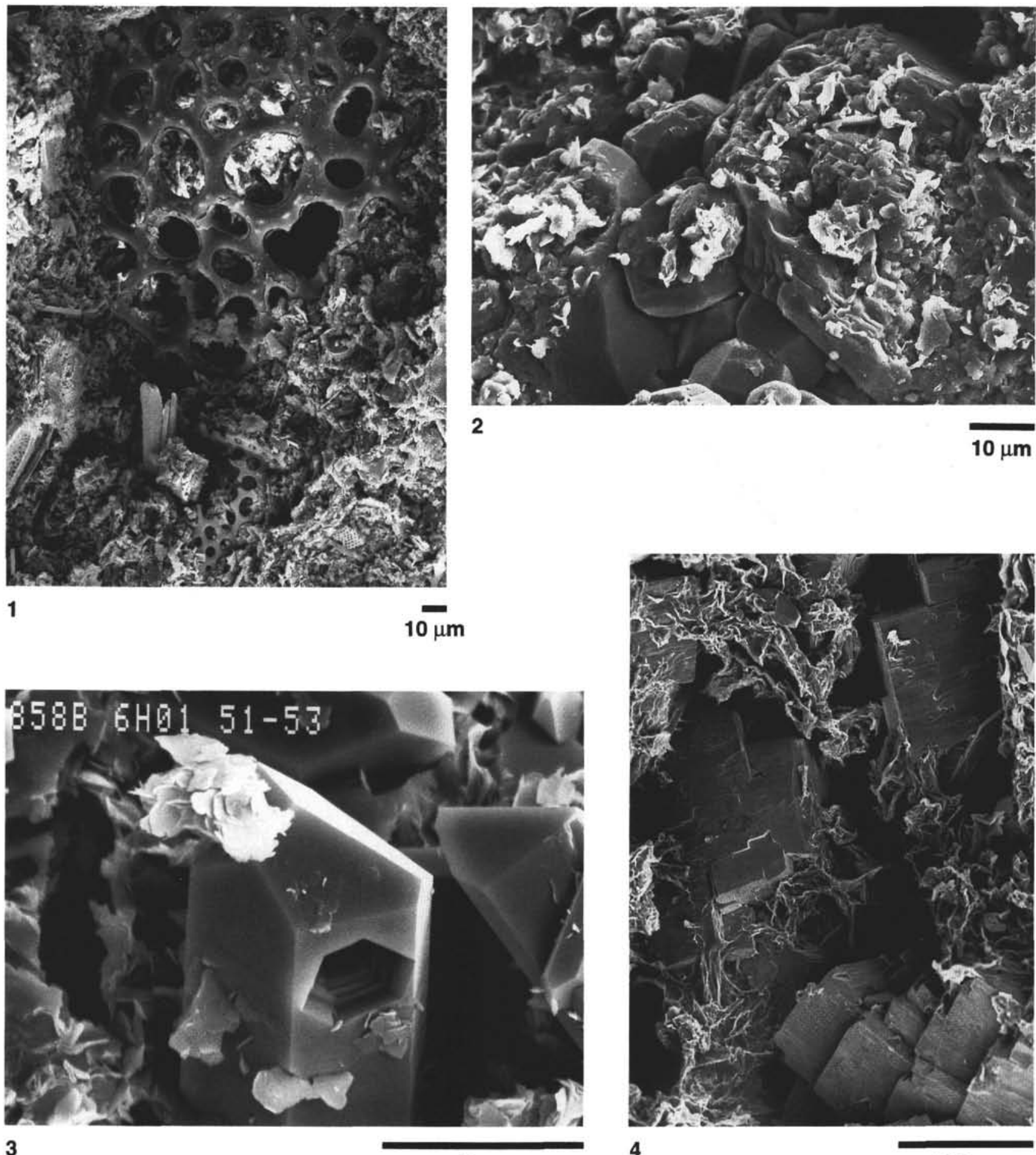

$10 \mu \mathrm{m}$

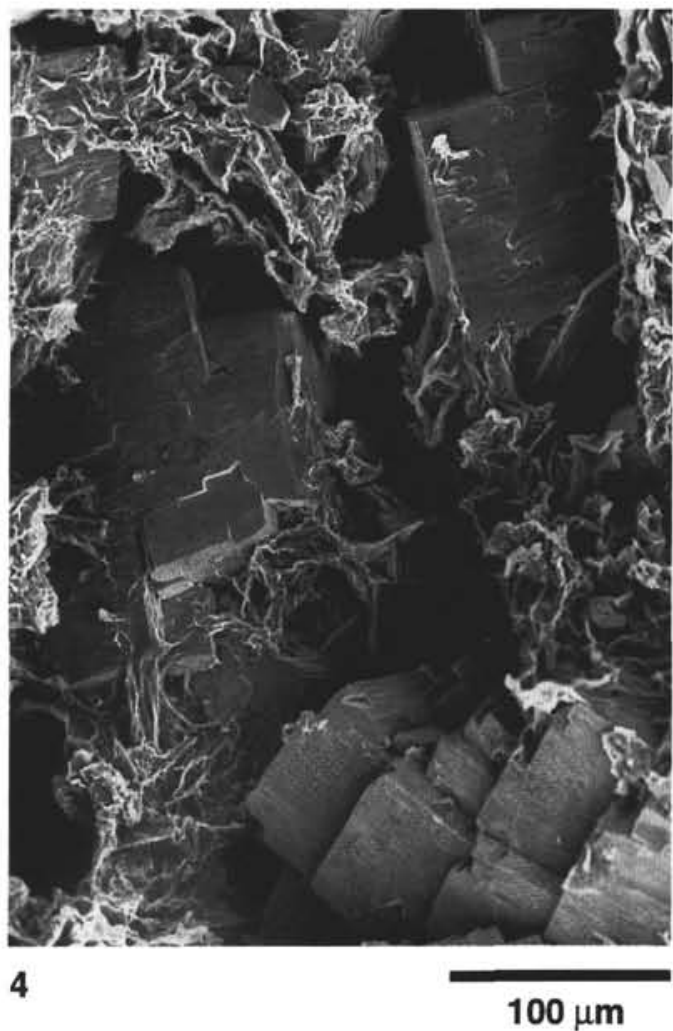

Plate 1. SEM photographs of fragments of bulk samples. 1. Typical texture from Unit I; mixture of detrital silicate with biogenic components is visible on this photograph of Sample 139-856A-1H-1, 48-51 cm. 2. Detrital, corroded quartz showing rounded edges and dissolution features in Sample 139-856A$6 \mathrm{H}-7,49-52 \mathrm{~cm}$ (Unit II). 3. Neoformed chlorite and quartz in Sample 139-858B-6H-1, 51-53 cm. 4. Neoformed pyrite and smectite in Sample $139-858 \mathrm{~B}-2 \mathrm{H}-3,75-77 \mathrm{~cm}$. 


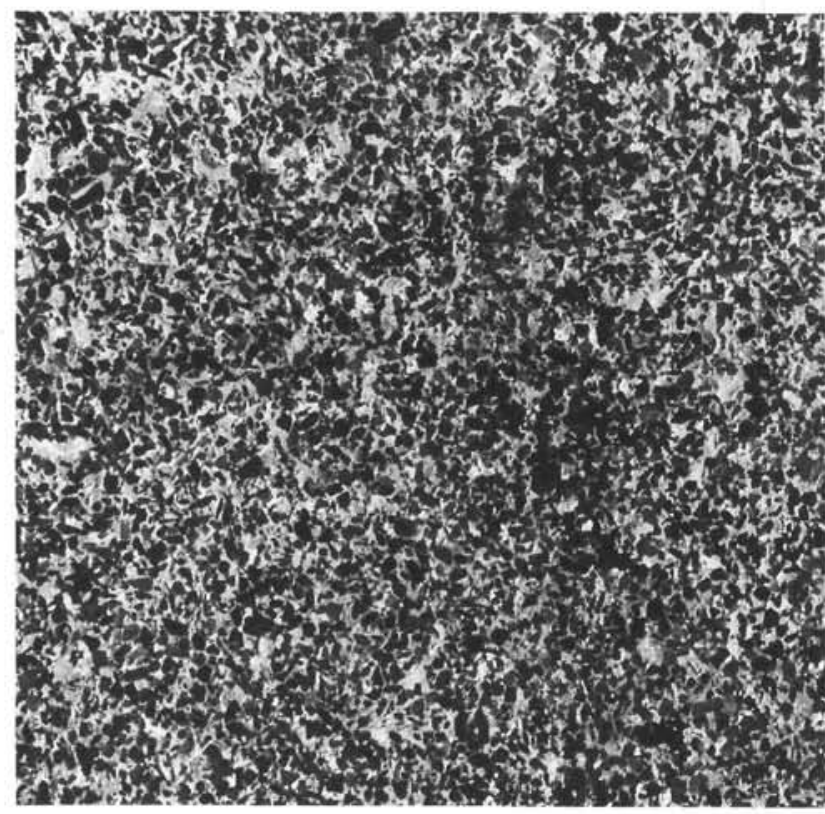

1

$0.25 \mu \mathrm{m}$

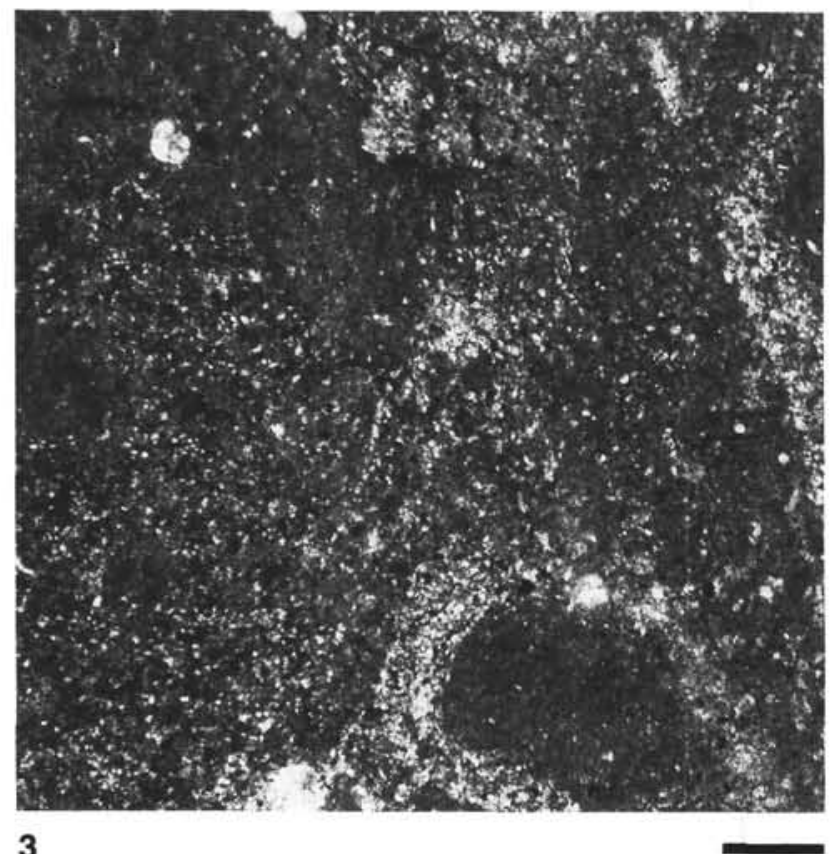

$0.25 \mu \mathrm{m}$

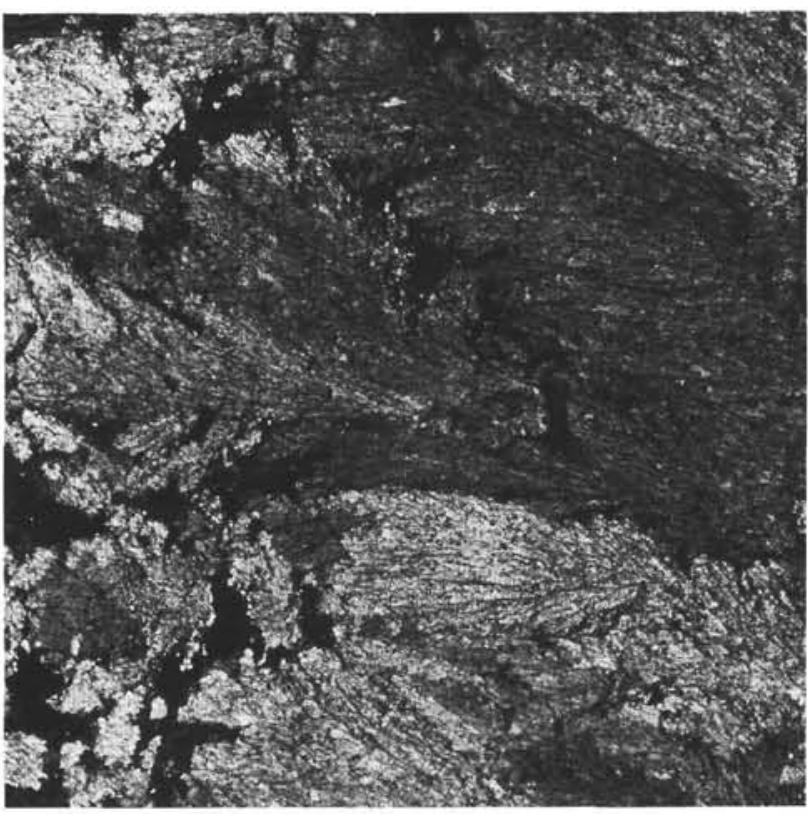

2

$0.25 \mu \mathrm{m}$

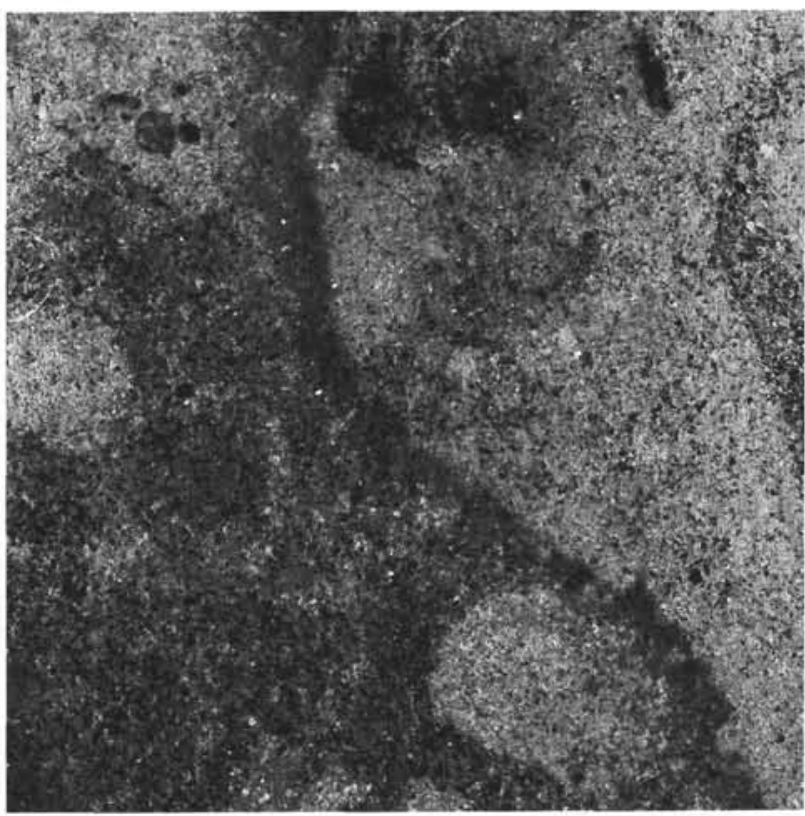

4

$0.5 \mu \mathrm{m}$

Plate 2. Cathodoluminescence and optical microscope photographs of carbonate cements and nodules. 1. Sample 139-856A-7H-CC, 38-42. Under cathodic light, the red orange (white in this photograph) network of unzoned Mn-bearing carbonate cementing the silt is visible. 2. Sample 139-856A-10X-2, 13-15 $\mathrm{cm}$. Calcite vein with plumose fabric and undulose extinction, cutting a carbonate-cemented silty nodule (optical microscopy). 3. Sample 139-858A-3H-1, 0-3 cm. Optical microscopy of silty clay-rich nodule irregularly impregnated by carbonate (burrowing traces?). 4. Sample $139-858 \mathrm{~A}-3 \mathrm{H}-1,0-3 \mathrm{~cm}$. Cathodoluminescence pattern on the same section as Figure 3 shows an orange carbonate following diagenetic fronts that possibly correspond to organic (?) structures in the nodule. 


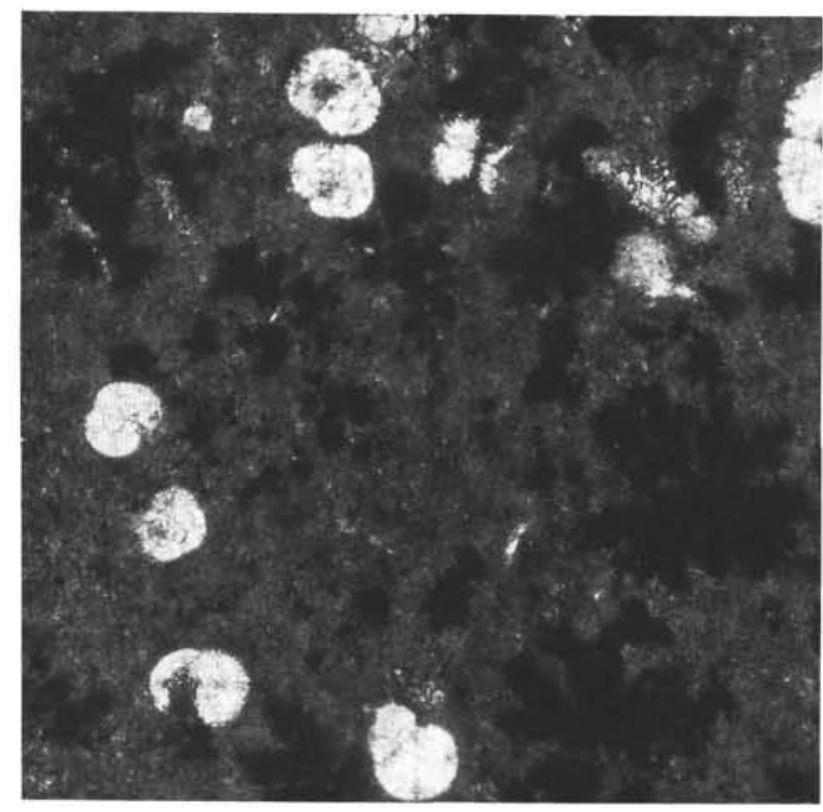

1

$\overline{0.25 \mu \mathrm{m}}$

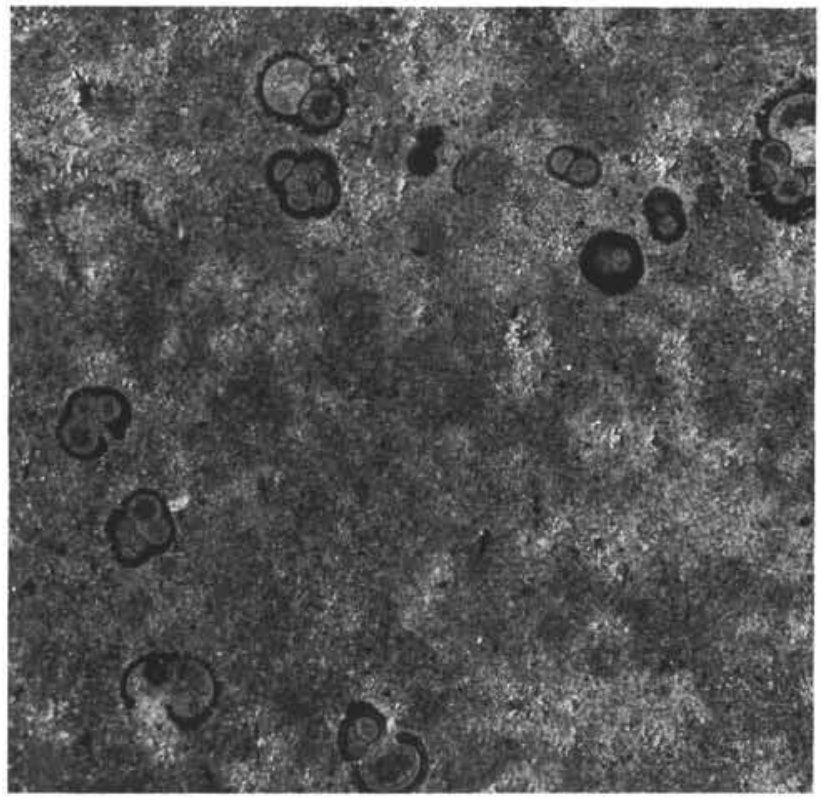

2

$0.25 \mu \mathrm{m}$
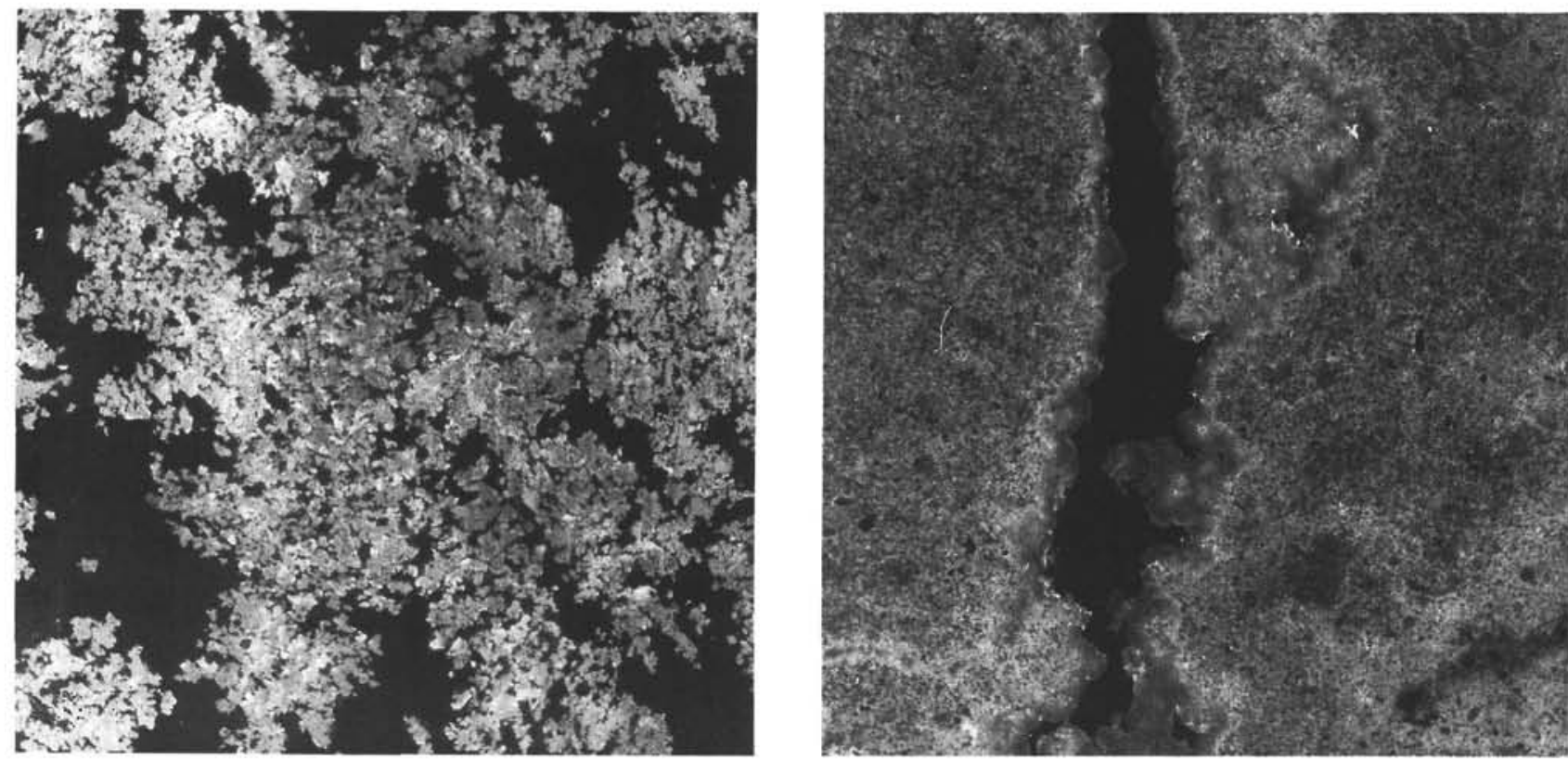

3

$0.25 \mu \mathrm{m}$

4

$0.25 \mu \mathrm{m}$

Plate 3. 1. Sample 139-858A-5H-06, 40-45 cm. Optical microphotograph of a calcite-cemented silty nodule with foraminifers. 2. Sample 139-858-5H-6, $40-45 \mathrm{~cm}$. Cathodoluminescence image of the same section as Figure 1; the Mn-devoid foraminifers are not luminescing, whereas most of the nodule sediment, cemented by Mn-bearing calcite, shows a patchy, bright-orange luminescence. 3. Sample 139-858D-1H-5, 75-77 cm. Arrow-shaped concretions of Mn-bearing calcite (bright red under cathodic light) cement a silty nodule. 4. Sample 139-858D-1H-1, 72-75 cm. Both the groundmass and the crystals filling the calcite veins belong to the same generation of low-Mn calcite (pale orange under cathodic light), patchily distributed throughout the nodule. 

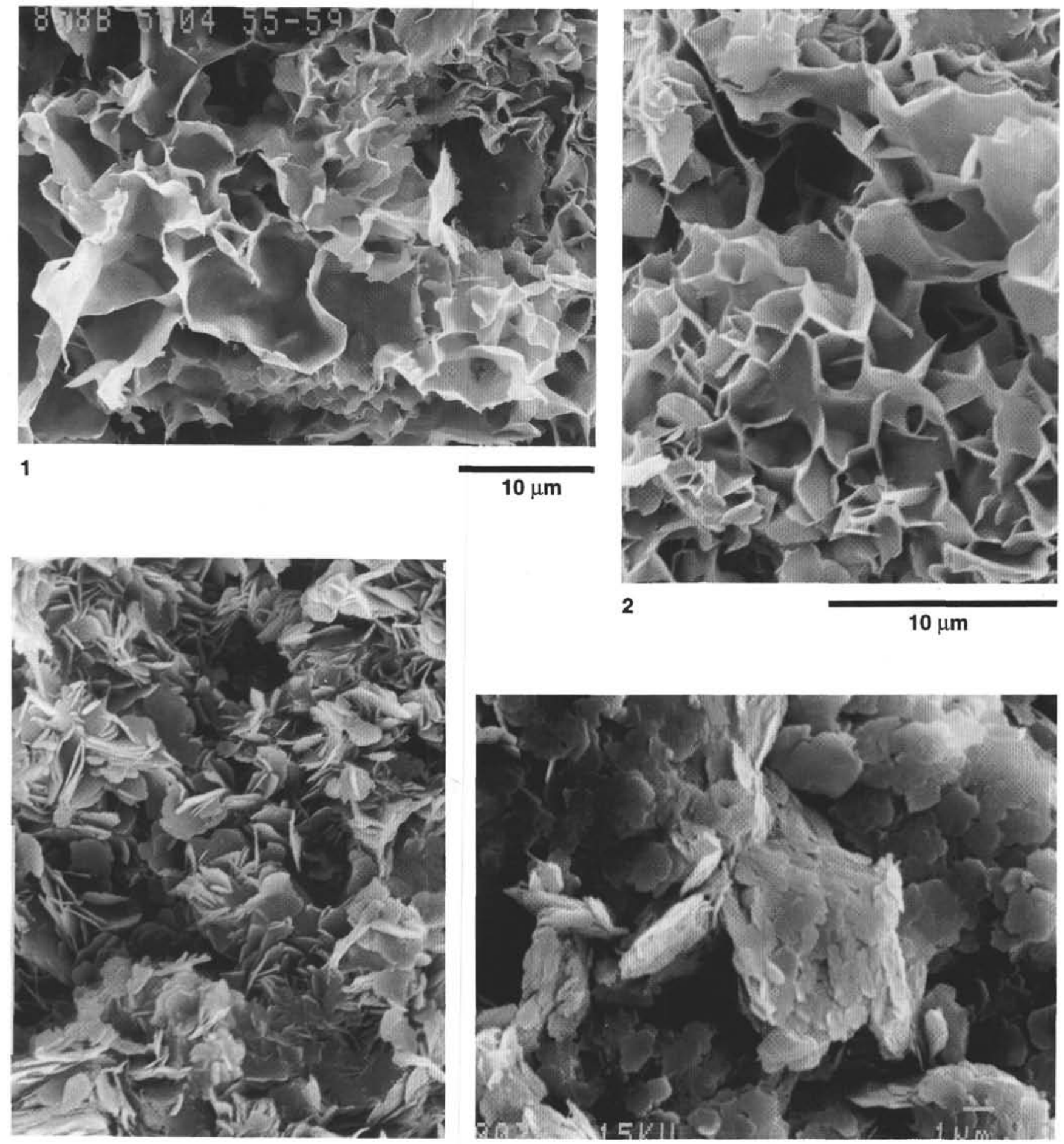

3

$10 \mu \mathrm{m}$

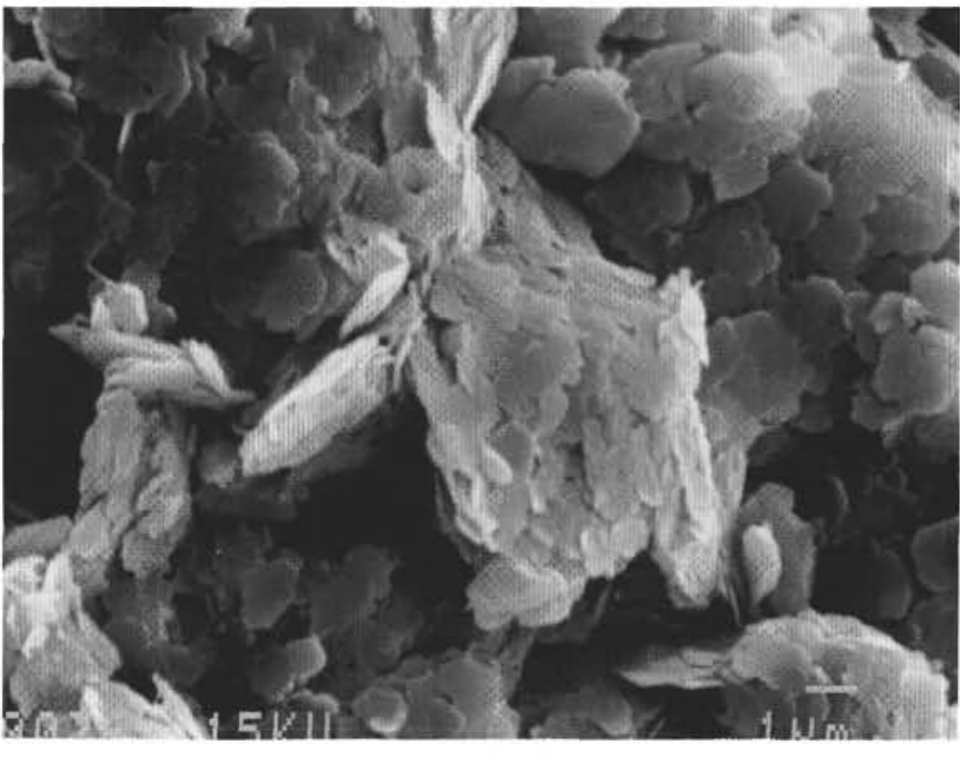

4

$10 \mu \mathrm{m}$

Plate 4. SEM photographs of bulk-sediment fragments of Hole 858B. 1. 139-858B-5H-4, 54-59 cm. Corrensite minerals showing typical corn-flake morphology. 2. Corrensite particles observed in Sample 139-858B-8X-2, 26-28 cm. 3. Sample 139-858B-8X-2, 26-28 cm. Chlorite minerals with rigid platelet morphology. 4. Sample 139-858B-6H-1,51-53 cm. Chlorite minerals showing typical rigid platelet morphology. 


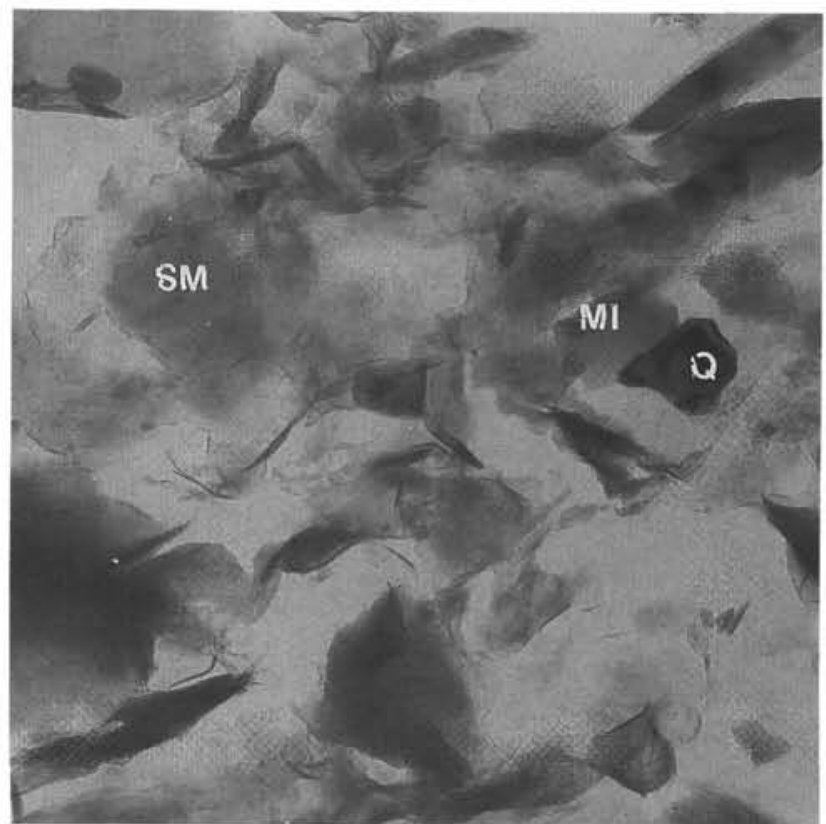

1
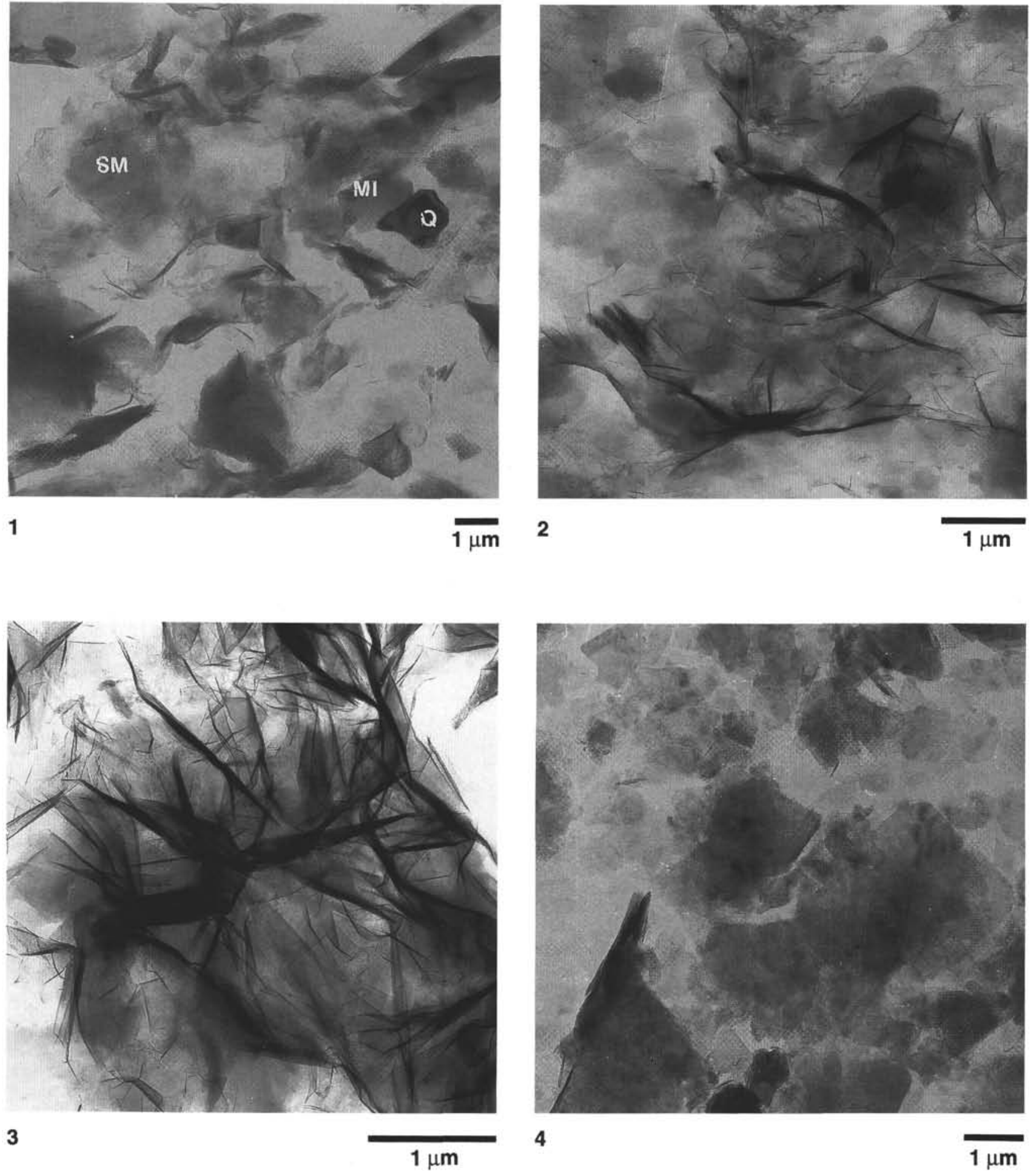

Plate 5. TEM photographs of the clay fraction of samples from Site 858. 1. Clay fraction of Sample 139-858B-1H-1, 48-51 cm (Unit I) shows several detrital particles with various morphologies and sizes. 2. Hydrothermal smectite particles with curled edges in Sample 139-858B-2H-3,75-77 cm. 3. Same hydrothermal smectite as in Figure 2 but at a higher magnification. 4. Hydrothermal chlorite particles with platelet morphology and mottled contrast (Sample 139-858B-6H-1, 51-53 cm). 